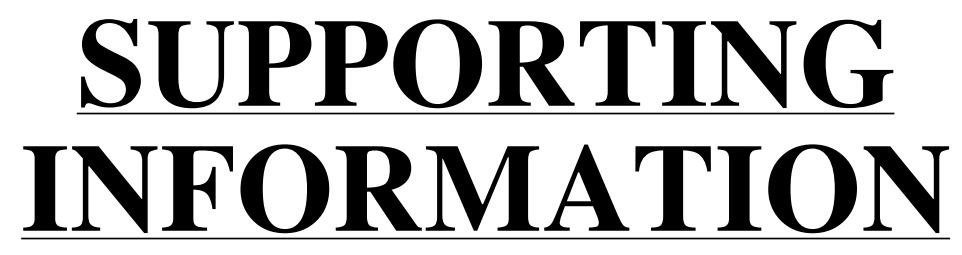

for
Shultz, Fico, Lee, Kampf, Kirschbaum, Pinkerton, and Boyle,"Mechanisms of Exchange Modulation in Trimethylenemethane-Type Biradicals: The Roles of Conformation and Spin Density"

Submitted to: Journal of the American Chemical Society 
Crystal Magnetic Susceptibility Data. Fit parameters $\boldsymbol{J}$ and $z J^{\prime}$ are in units of $\mathrm{cm}^{-1}$. "c" is biradical mole fraction. Data have been corrected for paramagnetic impurities.
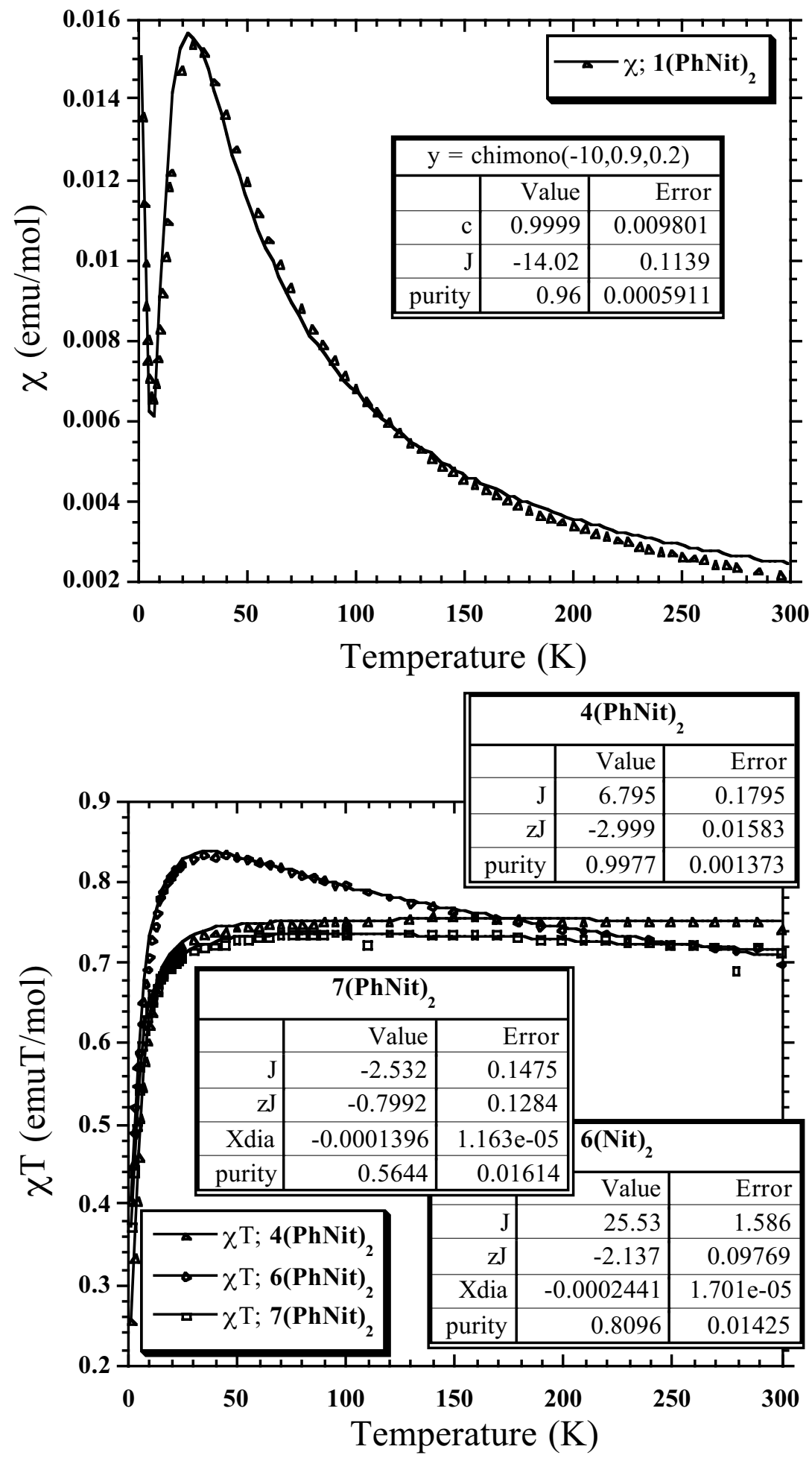


\section{Structure Determination.}

Red plates of $\mathbf{r f b i s}\left(\mathbf{1}\left(\mathbf{P h N i t}_{2}\right)\right)$ were crystallized from a diethyl ether/dichloromethane/heptane solution at $22 \mathrm{deg}$. C. A crystal of dimensions $0.34 \times 0.28 \times 0.08 \mathrm{~mm}$ was mounted on a standard Bruker SMART CCD-based $\mathrm{X}$-ray diffractometer equipped with a LT-2 low temperature device and normal focus Mo-target X-ray tube $(\lambda=$ $0.71073 \mathrm{~A})$ operated at $2000 \mathrm{~W}$ power $(50 \mathrm{kV}, 40 \mathrm{~mA})$. The X-ray intensities were measured at $118(2) \mathrm{K}$; the detector was placed at a distance $4.950 \mathrm{~cm}$ from the crystal. A total of 3862 frames were collected with a scan width of $0.2^{\circ}$ in $\omega$ with an exposure time of $30 \mathrm{~s} /$ frame. The frames were integrated with the Bruker SAINT software package with a narrow frame algorithm. The integration of the data yielded a total of 37135 reflections to a maximum $2 \theta$ value of $56.74^{\circ}$ of which 13853 were independent and 9779 were greater than $2 \sigma(\mathrm{I})$. The final cell constants (Table 1) were based on the xyz centroids of 5636 reflections above 10\%(I). Analysis of the data showed negligible decay during data collection; the data were processed with SADABS, no correction for absorption was necessary. The structure was solved and refined with the Bruker SHELXTL (version 5.10) software package, using the space group P1bar with $\mathrm{Z}=4$ for the formula $\mathrm{C}_{32} \mathrm{H}_{41} \mathrm{~N}_{2} \mathrm{O}_{2}$. There are two crystallographically independent molecules, one of which is partially disordered. All non-hydrogen atoms were refined anisotropically with the hydrogen atoms placed in idealized positions. Full matrix least-squares refinement based on $\mathrm{F}^{2}$ converged at $\mathrm{R} 1=$ 0.0742 and $w R 2=0.2060$ [based on $\mathrm{I}>2 \operatorname{sigma}(\mathrm{I})$ ], $\mathrm{R} 1=0.1045$ and $\mathrm{wR} 2=0.2282$ for all data. Additional details are presented in Table 1 and are given as Supporting Information in a CIF file.

Sheldrick, G.M. SHELXTL, v. 5.10; Bruker Analytical X-ray, Madison, WI, 1997.

Sheldrick, G.M. SADABS. Program for Empirical Absorption Correction of Area Detector Data, University of Gottingen: Gottingen, Germany, 1996.

Saint Plus, v. 6.29, Bruker Analytical X-ray, Madison, WI, 2001.

Table S1. Crystal data and structure refinement for rfbis $\left(\mathbf{1}\left(\mathbf{P h N i t}_{2}\right)\right)$.

Identification code

Empirical formula

rfbis

Formula weight

C32 H41 N2 O2

Temperature

485.67

Wavelength

$118(2) \mathrm{K}$

Crystal system, space group

$0.71073 \mathrm{~A}$

Unit cell dimensions

Triclinic, P-1

$\mathrm{a}=12.865(3) \mathrm{A}$; alpha $=108.424(3) \mathrm{deg}$.

$\mathrm{b}=14.483(3) \mathrm{A}$; beta $=91.425(4)$ deg.

$\mathrm{c}=16.875(4) \mathrm{A} ;$ gamma $=108.159(3) \mathrm{deg}$.

Volume 2807.8(10) $\mathrm{A}^{\wedge} 3$

Z, Calculated density

4, $1.149 \mathrm{Mg} / \mathrm{m}^{\wedge} 3$

Absorption coefficient

$\mathrm{F}(000)$

$0.071 \mathrm{~mm}^{\wedge}-1$

Crystal size

Theta range for data collection

1052

$0.34 \times 0.28 \times 0.08 \mathrm{~mm}$

2.66 to 28.49 deg.

$-17<=\mathrm{h}<=17,-19<=\mathrm{k}<=18,-22<=\mathrm{l}<=22$

Limiting indices

$13853 / 13853$ [R(int) $=0.0356]$

$28.49 \quad 97.2 \%$

None

0.9943 and 0.9763

Absorption correction

Max. and min. transmission

Refinement method

Data / restraints / parameters

Goodness-of-fit on $\mathrm{F}^{\wedge} 2$

Full-matrix least-squares on $\mathrm{F}^{\wedge} 2$

13853 / 0 / 766

1.033

Final $\mathrm{R}$ indices [ $\mathrm{I}>2 \operatorname{sigma}(\mathrm{I})]$

$\mathrm{R}$ indices (all data)

Largest diff. peak and hole

$\mathrm{R} 1=0.0742, \mathrm{wR} 2=0.2060$

$\mathrm{R} 1=0.1045, \mathrm{wR} 2=0.2282$

0.735 and -0.579 e. $\mathrm{A}^{\wedge}-3$

Table S2. Atomic coordinates ( $\left.\times 10^{\wedge} 4\right)$ and equivalent isotropic displacement parameters $\left(\mathrm{A}^{\wedge} 2 \times 10^{\wedge} 3\right.$ ) for rfbis $\left(\mathbf{1}\left(\mathbf{P h N i t}_{2}\right)\right)$. U(eq) is defined as one third of the trace of the orthogonalized Uij tensor.

\begin{tabular}{ll}
\hline $\mathrm{x} \mathrm{y} \mathrm{z}$ & $\mathrm{U}(\mathrm{eq})$ \\
\hline $\mathrm{C}(1)$ & $3176(2) 1809(2) 4296(1) 20(1)$ \\
$\mathrm{C}(2)$ & $4370(2) 1905(2) 4395(1) 20(1)$ \\
$\mathrm{C}(3)$ & $5175(2) 2581(2) 4114(1) 21(1)$ \\
$\mathrm{C}(4)$ & $6278(2) 2658(2) 4209(1) 23(1)$ \\
$\mathrm{C}(5)$ & $6598(2) 2051(2) 4599(1) 24(1)$
\end{tabular}


C(6) 5808(2) 1384(2) 4894(1) 27(1)

C(7) 4711(2) 1316(2) 4798(1) 25(1)

C(8) 8725(2) 2914(2) 4697(2) 32(1)

C(9) 8636(2) 3943(2) 5248(2) 39(1)

C(10) 9682(2) 2735(2) 5107(2) 45(1)

C(11) 8913(2) 2886(3) 3803(2) 47(1)

C(12) 2915(2) 2624(2) 4983(1) 21(1)

C(13) 3250(2) 3645(2) 4994(1) 29(1)

C(14) 3096(2) 4429(2) 5651(1) 29(1)

C(15) 2585(2) 4215(2) 6328(1) 23(1)

C(16) 2225(2) 3194(2) 6313(1) 23(1)

C(17) 2395(2) 2419(2) 5653(1) 21(1)

C(18) 1938(2) 4996(2) 7763(1) 25(1)

C(19) 2034(2) 6091(2) 8294(2) 36(1)

C(20) 2550(2) 4582(2) 8284(2) 33(1)

C(21) 722(2) 4342(2) 7498(2) 36(1)

C(22) 2434(2) 1065(2) 3660(1) 21(1)

C(23) 1225(2) 971(2) 3608(1) 28(1)

C(24) 702(2) 851(2) 2735(2) 36(1)

$\mathrm{C}(25)$ 1456(2) 1467(2) 2261(2) 40(1)

C(26) 2197(2) 920(2) 1755(2) 36(1)

C(27) 3126(2) 816(2) 2258(1) 29(1)

C(28) 2816(2) 341(2) 2955(1) 23(1)

C(29) 2047(2) -790(2) 2604(1) 30(1)

C(30) 1468(2)-1222(2) 3256(2) 37(1)

C(31) 405(2) -987(2) 3440(2) 41(1)

C(32) 537(2) 134(2) 3945(2) 39(1)

C(33) 4941(2) 8000(2)10650(1) 21(1)

C(34) 3719(2) 7785(2)10625(1) 22(1)

C(35) 3228(2) 8394(2)10366(1) 24(1)

C(36) 2103(2) 8204(2)10322(1) 26(1)

C(37) 1434(2) 7396(2)10544(1) 30(1)

C(38) 1912(2) 6777(2)10803(2) 34(1)

C(39) 3036(2) 6966(2)10836(2) 29(1)

C(40) $-467(2)$ 7368(2) 9969(2) 39(1)

C(41) $-336(3)$ 8543(3)10256(2) 55(1)

C(42) -1644(2) 6781(3)10028(2) 57(1)

C(43) -208(2) 7027(2) 9079(2) 42(1)

C(44) 5258(12)7249(11)9991(8) 22(2)

C(45) 5226(5) 7258(4) 9164(4) 30(1)

C(46) 5380(6) 6474(5) 8481(4) 35(1)

C(47) 5595(5) 5663(5) 8636(4) 27(1)

C(48) 5646(4) 5629(3) 9461(3) 25(1)

C(49) 5479(4) 6406(4)10103(4) 24(1)

C(50) 6337(7) 4755(5) 7283(5) 25(2)

C(51) 7295(12)4484(14)7591(11) 118(7)

C(52) 6949(9) 5803(5) 7217(5) 89(3)

C(53) 5710(8) 3930(8) 6461(5) 92(3)

$\mathrm{O}(4) \quad 5265(5)$ 3899(3) 8113(3) 65(1)

$\mathrm{N}(4)$ 5666(4) 4775(3) 7983(3) 43(1)

C(44A) 5192(11)7357(10)9795(6) 16(2)

C(45A) 5540(4) 6550(4) 9738(3) 21(1)

C(46A) 5760(5) 5968(4) 8971(4) 21(1)

C(47A) 5635(4) 6219(4) 8254(3) 20(1)

C(48A) 5238(4) 7035(3) 8310(3) 25(1)

C(49A) 5023(4) 7589(4) 9074(3) 25(1)

C(50A) 6096(6) 4724(6) 7170(4) 18(2)

C(51A) 6170(5) 4395(4) 6230(3) 36(1) 
C(52A) 5178(4) 3901(3) 7373(3) 32(1) C(53A) 7215(7) 4849(6) 7609(5) 37(2)

$\mathrm{N}(4 \mathrm{~A})$ 5897(3) 5725(3) 7451(2) 25(1)

$\mathrm{O}(4 \mathrm{~A})$ 5963(4) 6181(3) 6907(2) 46(1)

C(54) 5685(2) 8736(2)11291(1) 20(1)

C(55) 5297(2) 9312(2)12080(1) 25(1)

C(56) 5216(2) 8760(2)12730(1) 33(1)

C(57) 6304(2) 8757(2)13121(2) 37(1)

C(58) 7051(2) 8376(2)12509(2) 34(1)

C(59) 7605(2) 9107(2)12042(1) 27(1)

C(60) 6911(2) 8985(2)11239(1) 22(1)

C(61) 7358(2) 9914(2)10932(1) 27(1)

C(62) 7389(2)10967(2)11527(2) 32(1)

C(63) 6298(2)11031(2)11842(2) 34(1)

C(64) 5937(2)10474(2)12469(1) 30(1)

$\mathrm{N}(1)$ 7698(2) 2039(2) 4662(1) 31(1)

$\mathrm{N}(2) \quad 2485(2)$ 5058(1) 6993(1) 31(1)

$\mathrm{N}(3) \quad 282(2) 7207(2) 10561(1) 44(1)$

$\mathrm{O}(1)$ 7820(2) 1195(2) 4687(1) 50(1)

$\mathrm{O}(2) \quad 2902(3)$ 5956(2) 6937(1) 72(1)

$\mathrm{O}(3)-151(2)$ 6794(3)11082(2)100(1)

Table S3. Bond lengths [A] and angles [deg] for $\mathbf{r b b i s}\left(\mathbf{1}(\mathbf{P h N i t})_{2}\right)$.

\begin{tabular}{ll}
\hline $\mathrm{C}(1)-\mathrm{C}(22)$ & $1.342(3)$ \\
$\mathrm{C}(1)-\mathrm{C}(2)$ & $1.499(3)$ \\
$\mathrm{C}(1)-\mathrm{C}(12)$ & $1.500(3)$ \\
$\mathrm{C}(2)-\mathrm{C}(3)$ & $1.393(3)$ \\
$\mathrm{C}(2)-\mathrm{C}(7)$ & $1.403(3)$ \\
$\mathrm{C}(3)-\mathrm{C}(4)$ & $1.389(3)$ \\
$\mathrm{C}(4)-\mathrm{C}(5)$ & $1.398(3)$ \\
$\mathrm{C}(5)-\mathrm{C}(6)$ & $1.390(3)$ \\
$\mathrm{C}(5)-\mathrm{N}(1)$ & $1.422(3)$ \\
$\mathrm{C}(6)-\mathrm{C}(7)$ & $1.385(3)$ \\
$\mathrm{C}(8)-\mathrm{N}(1)$ & $1.504(3)$ \\
$\mathrm{C}(8)-\mathrm{C}(11)$ & $1.523(4)$ \\
$\mathrm{C}(8)-\mathrm{C}(10)$ & $1.527(3)$ \\
$\mathrm{C}(8)-\mathrm{C}(9)$ & $1.528(3)$ \\
$\mathrm{C}(12)-\mathrm{C}(17)$ & $1.393(3)$ \\
$\mathrm{C}(12)-\mathrm{C}(13)$ & $1.399(3)$ \\
$\mathrm{C}(13)-\mathrm{C}(14)$ & $1.383(3)$ \\
$\mathrm{C}(14)-\mathrm{C}(15)$ & $1.402(3)$ \\
$\mathrm{C}(15)-\mathrm{C}(16)$ & $1.397(3)$ \\
$\mathrm{C}(15)-\mathrm{N}(2)$ & $1.416(3)$ \\
$\mathrm{C}(16)-\mathrm{C}(17)$ & $1.386(3)$ \\
$\mathrm{C}(18)-\mathrm{N}(2)$ & $1.507(3)$ \\
$\mathrm{C}(18)-\mathrm{C}(19)$ & $1.519(3)$ \\
$\mathrm{C}(18)-\mathrm{C}(21)$ & $1.523(3)$ \\
$\mathrm{C}(18)-\mathrm{C}(20)$ & $1.530(3)$ \\
$\mathrm{C}(22)-\mathrm{C}(23)$ & $1.516(3)$ \\
$\mathrm{C}(22)-\mathrm{C}(28)$ & $1.523(3)$ \\
$\mathrm{C}(23)-\mathrm{C}(24)$ & $1.538(3)$ \\
$\mathrm{C}(23)-\mathrm{C}(32)$ & $1.539(3)$ \\
$\mathrm{C}(24)-\mathrm{C}(25)$ & $1.525(4)$ \\
$\mathrm{C}(25)-\mathrm{C}(26)$ & $1.532(4)$ \\
$\mathrm{C}(26)-\mathrm{C}(27)$ & $1.521(3)$ \\
$\mathrm{C}(27)-\mathrm{C}(28)$ & $1.543(3)$ \\
$\mathrm{C}(28)-\mathrm{C}(29)$ & $1.537(3)$
\end{tabular}


$\mathrm{C}(29)-\mathrm{C}(30) 1.531(3)$

$\mathrm{C}(30)-\mathrm{C}(31) 1.525(4)$

$\mathrm{C}(31)-\mathrm{C}(32) 1.529(4)$

$\mathrm{C}(33)-\mathrm{C}(54) 1.344(3)$

C(33)-C(44) 1.456(17)

C(33)-C(34) 1.500(3)

C(33)-C(44A)1.554(13)

C(34)-C(39) 1.392(3)

$\mathrm{C}(34)-\mathrm{C}(35) 1.395(3)$

$\mathrm{C}(35)-\mathrm{C}(36) 1.382(3)$

C(36)-C(37) 1.384(3)

C(37)-C(38) 1.397(4)

$\mathrm{C}(37)-\mathrm{N}(3)$ 1.423(3)

C(38)-C(39) 1.381(3)

C(40)-N(3) 1.491(3)

$\mathrm{C}(40)-\mathrm{C}(43) 1.506(4)$

C(40)-C(42) 1.510(4)

C(40)-C(41) 1.567(4)

C(44)-C(45) 1.399(9)

C(44)-C(49) 1.402(13)

$\mathrm{C}(45)-\mathrm{C}(46) 1.407(9)$

$\mathrm{C}(46)-\mathrm{C}(47) 1.384(9)$

C(47)-C(48) 1.409(7)

C(47)-N(4) 1.436(7)

$\mathrm{C}(48)-\mathrm{C}(49) 1.371(6)$

$\mathrm{C}(50)-\mathrm{N}(4)$ 1.478(9)

$\mathrm{C}(50)-\mathrm{C}(53) 1.517(5)$

C(50)-C(52) 1.519(5)

$\mathrm{C}(50)-\mathrm{C}(51) 1.530(5)$

$\mathrm{O}(4)-\mathrm{N}(4) \quad 1.302(6)$

C(44A)-C(45A) 1.354(14)

C(44A)-C(49A) 1.389(8)

C(45A)-C(46A) $1.395(7)$

C(46A)-C(47A) 1.388(8)

$\mathrm{C}(47 \mathrm{~A})-\mathrm{C}(48 \mathrm{~A}) \quad 1.405(6)$

$\mathrm{C}(47 \mathrm{~A})-\mathrm{N}(4 \mathrm{~A}) 1.423(6)$

C(48A)-C(49A) 1.373(6)

$\mathrm{C}(50 \mathrm{~A})-\mathrm{N}(4 \mathrm{~A}) 1.482(10)$

$\mathrm{C}(50 \mathrm{~A})-\mathrm{C}(51 \mathrm{~A}) \quad 1.521(5)$

$\mathrm{C}(50 \mathrm{~A})-\mathrm{C}(52 \mathrm{~A}) \quad 1.528(5)$

$\mathrm{C}(50 \mathrm{~A})-\mathrm{C}(53 \mathrm{~A}) \quad 1.534(5)$

$\mathrm{N}(4 \mathrm{~A})-\mathrm{O}(4 \mathrm{~A}) 1.279(5)$

$\mathrm{C}(54)-\mathrm{C}(60) 1.516(3)$

C(54)-C(55) 1.520(3)

$\mathrm{C}(55)-\mathrm{C}(64) 1.533(3)$

$\mathrm{C}(55)-\mathrm{C}(56) 1.537(3)$

$\mathrm{C}(56)-\mathrm{C}(57)$ 1.534(3)

$\mathrm{C}(57)-\mathrm{C}(58) 1.521(4)$

$\mathrm{C}(58)-\mathrm{C}(59) 1.528(3)$

$\mathrm{C}(59)-\mathrm{C}(60) 1.534(3)$

$\mathrm{C}(60)-\mathrm{C}(61) 1.545(3)$

C(61)-C(62) 1.526(3)

C(62)-C(63) 1.532(3)

C(63)-C(64) 1.522(3)

$\mathrm{N}(1)-\mathrm{O}(1) \quad 1.293(3)$

$\mathrm{N}(2)-\mathrm{O}(2) \quad 1.278(3)$

$\mathrm{N}(3)-\mathrm{O}(3) \quad 1.261(3)$

C(22)-C(1)-C(2) 122.81(18) 
C(22)-C(1)-C(12)124.57(18)

$\mathrm{C}(2)-\mathrm{C}(1)-\mathrm{C}(12)$ 112.62(17)

C(3)-C(2)-C(7) 117.80(19)

$\mathrm{C}(3)-\mathrm{C}(2)-\mathrm{C}(1)$ 122.34(18)

$\mathrm{C}(7)-\mathrm{C}(2)-\mathrm{C}(1)$ 119.85(18)

$\mathrm{C}(4)-\mathrm{C}(3)-\mathrm{C}(2) \quad 121.61(19)$

C(3)-C(4)-C(5) 119.68(19)

$\mathrm{C}(6)-\mathrm{C}(5)-\mathrm{C}(4) \quad 119.5(2)$

$\mathrm{C}(6)-\mathrm{C}(5)-\mathrm{N}(1) \quad 118.0(2)$

$\mathrm{C}(4)-\mathrm{C}(5)-\mathrm{N}(1) \quad 122.4(2)$

$\mathrm{C}(7)-\mathrm{C}(6)-\mathrm{C}(5) \quad 120.3(2)$

$\mathrm{C}(6)-\mathrm{C}(7)-\mathrm{C}(2)$ 121.1(2)

$\mathrm{N}(1)-\mathrm{C}(8)-\mathrm{C}(11) 109.2(2)$

$\mathrm{N}(1)-\mathrm{C}(8)-\mathrm{C}(10) 106.8(2)$

C(11)-C(8)-C(10)109.4(2)

$\mathrm{N}(1)-\mathrm{C}(8)-\mathrm{C}(9) \quad 110.20(18)$

$\mathrm{C}(11)-\mathrm{C}(8)-\mathrm{C}(9) 112.6(2)$

$\mathrm{C}(10)-\mathrm{C}(8)-\mathrm{C}(9) 108.5(2)$

$\mathrm{C}(17)-\mathrm{C}(12)-\mathrm{C}(13) \quad 117.36(18)$

$\mathrm{C}(17)-\mathrm{C}(12)-\mathrm{C}(1) 122.32(18)$

C(13)-C(12)-C(1)120.22(18)

C(14)-C(13)-C(12) 121.7(2)

C(13)-C(14)-C(15) 120.4(2)

$\mathrm{C}(16)-\mathrm{C}(15)-\mathrm{C}(14) \quad 118.36(19)$

$\mathrm{C}(16)-\mathrm{C}(15)-\mathrm{N}(2) 124.11(19)$

$\mathrm{C}(14)-\mathrm{C}(15)-\mathrm{N}(2) 117.53(19)$

C(17)-C(16)-C(15) 120.49(19)

C(16)-C(17)-C(12) 121.71(19)

$\mathrm{N}(2)-\mathrm{C}(18)-\mathrm{C}(19) 107.02(18)$

$\mathrm{N}(2)-\mathrm{C}(18)-\mathrm{C}(21) 109.63(19)$

$\mathrm{C}(19)-\mathrm{C}(18)-\mathrm{C}(21) \quad 109.11(19)$

$\mathrm{N}(2)-\mathrm{C}(18)-\mathrm{C}(20) 110.14(18)$

$\mathrm{C}(19)-\mathrm{C}(18)-\mathrm{C}(20) \quad 108.11(19)$

$\mathrm{C}(21)-\mathrm{C}(18)-\mathrm{C}(20) \quad 112.66(19)$

$\mathrm{C}(1)-\mathrm{C}(22)-\mathrm{C}(23) 120.87(18)$

$\mathrm{C}(1)-\mathrm{C}(22)-\mathrm{C}(28) 119.76(18)$

$\mathrm{C}(23)-\mathrm{C}(22)-\mathrm{C}(28) \quad 119.29(17)$

$\mathrm{C}(22)-\mathrm{C}(23)-\mathrm{C}(24) \quad 114.35(18)$

$\mathrm{C}(22)-\mathrm{C}(23)-\mathrm{C}(32) \quad 112.1(2)$

C(24)-C(23)-C(32) 112.0(2)

$\mathrm{C}(25)-\mathrm{C}(24)-\mathrm{C}(23) \quad 115.3(2)$

$\mathrm{C}(24)-\mathrm{C}(25)-\mathrm{C}(26) \quad 114.2(2)$

$\mathrm{C}(27)-\mathrm{C}(26)-\mathrm{C}(25) \quad 116.8(2)$

$\mathrm{C}(26)-\mathrm{C}(27)-\mathrm{C}(28) \quad 117.36(19)$

$\mathrm{C}(22)-\mathrm{C}(28)-\mathrm{C}(29) \quad 116.14(17)$

$\mathrm{C}(22)-\mathrm{C}(28)-\mathrm{C}(27) \quad 109.71(17)$

$\mathrm{C}(29)-\mathrm{C}(28)-\mathrm{C}(27) \quad 112.61(18)$

$\mathrm{C}(30)-\mathrm{C}(29)-\mathrm{C}(28) \quad 115.24(19)$

$\mathrm{C}(31)-\mathrm{C}(30)-\mathrm{C}(29) \quad 113.9(2)$

C(30)-C(31)-C(32) 116.4(2)

$\mathrm{C}(31)-\mathrm{C}(32)-\mathrm{C}(23) \quad 117.1(2)$

$\mathrm{C}(54)-\mathrm{C}(33)-\mathrm{C}(44) \quad 121.4(6)$

$\mathrm{C}(54)-\mathrm{C}(33)-\mathrm{C}(34) \quad 123.15(18)$

$\mathrm{C}(44)-\mathrm{C}(33)-\mathrm{C}(34) \quad 114.8(6)$

$\mathrm{C}(54)-\mathrm{C}(33)-\mathrm{C}(44 \mathrm{~A}) \quad 126.6(5)$

$\mathrm{C}(44)-\mathrm{C}(33)-\mathrm{C}(44 \mathrm{~A}) \quad 15.8(5)$

C(34)-C(33)-C(44A) 109.8(5)

C(39)-C(34)-C(35) 117.7(2) 


\begin{tabular}{|c|c|}
\hline $\mathrm{C}(39)-\mathrm{C}(34)-\mathrm{C}(33)$ & $121.84(19)$ \\
\hline $\mathrm{C}(35)-\mathrm{C}(34)-\mathrm{C}(33)$ & $120.40(18)$ \\
\hline $\mathrm{C}(36)-\mathrm{C}(35)-\mathrm{C}(34)$ & $121.8(2)$ \\
\hline $\mathrm{C}(35)-\mathrm{C}(36)-\mathrm{C}(37)$ & $120.0(2)$ \\
\hline $\mathrm{C}(36)-\mathrm{C}(37)-\mathrm{C}(38)$ & $119.0(2)$ \\
\hline \multicolumn{2}{|c|}{$\mathrm{C}(36)-\mathrm{C}(37)-\mathrm{N}(3) 122.2(2)$} \\
\hline \multicolumn{2}{|c|}{$\mathrm{C}(38)-\mathrm{C}(37)-\mathrm{N}(3) 118.7(2)$} \\
\hline $\mathrm{C}(39)-\mathrm{C}(38)-\mathrm{C}(37)$ & $120.5(2)$ \\
\hline $\mathrm{C}(38)-\mathrm{C}(39)-\mathrm{C}(34)$ & $121.0(2)$ \\
\hline \multicolumn{2}{|c|}{$\mathrm{N}(3)-\mathrm{C}(40)-\mathrm{C}(43) 111.4(2)$} \\
\hline \multicolumn{2}{|c|}{$\mathrm{N}(3)-\mathrm{C}(40)-\mathrm{C}(42) 108.3(2)$} \\
\hline $\mathrm{C}(43)-\mathrm{C}(40)-\mathrm{C}(42)$ & $111.3(2)$ \\
\hline \multicolumn{2}{|c|}{$\mathrm{N}(3)-\mathrm{C}(40)-\mathrm{C}(41) 109.1(2)$} \\
\hline $\mathrm{C}(43)-\mathrm{C}(40)-\mathrm{C}(41)$ & $109.2(2)$ \\
\hline $\mathrm{C}(42)-\mathrm{C}(40)-\mathrm{C}(41)$ & $107.5(2)$ \\
\hline $\mathrm{C}(45)-\mathrm{C}(44)-\mathrm{C}(49)$ & $115.3(12)$ \\
\hline$C(45)-C(44)-C(33)$ & $120.8(9)$ \\
\hline$C(49)-C(44)-C(33)$ & $123.5(7)$ \\
\hline $\mathrm{C}(44)-\mathrm{C}(45)-\mathrm{C}(46)$ & $123.3(9)$ \\
\hline $\mathrm{C}(47)-\mathrm{C}(46)-\mathrm{C}(45)$ & $118.5(6)$ \\
\hline $\mathrm{C}(46)-\mathrm{C}(47)-\mathrm{C}(48)$ & $120.1(5)$ \\
\hline \multicolumn{2}{|c|}{$\mathrm{C}(46)-\mathrm{C}(47)-\mathrm{N}(4) 123.5(6)$} \\
\hline \multicolumn{2}{|c|}{$\mathrm{C}(48)-\mathrm{C}(47)-\mathrm{N}(4) 116.0(5)$} \\
\hline C(49)-C(48)-C(47) & $119.1(5)$ \\
\hline $\mathrm{C}(48)-\mathrm{C}(49)-\mathrm{C}(44)$ & $123.7(7)$ \\
\hline \multicolumn{2}{|c|}{$\mathrm{N}(4)-\mathrm{C}(50)-\mathrm{C}(53) 112.0(7)$} \\
\hline \multicolumn{2}{|c|}{$\mathrm{N}(4)-\mathrm{C}(50)-\mathrm{C}(52) 115.3(6)$} \\
\hline $\mathrm{C}(53)-\mathrm{C}(50)-\mathrm{C}(52)$ & $114.9(8)$ \\
\hline \multicolumn{2}{|c|}{$\mathrm{N}(4)-\mathrm{C}(50)-\mathrm{C}(51) 101.8(8)$} \\
\hline$C(53)-C(50)-C(51)$ & $110.4(10)$ \\
\hline$C(52)-C(50)-C(51)$ & $100.9(9)$ \\
\hline \multicolumn{2}{|c|}{$\mathrm{O}(4)-\mathrm{N}(4)-\mathrm{C}(47) 115.5(4)$} \\
\hline \multicolumn{2}{|c|}{$\mathrm{O}(4)-\mathrm{N}(4)-\mathrm{C}(50) 115.3(5)$} \\
\hline \multicolumn{2}{|c|}{$\mathrm{C}(47)-\mathrm{N}(4)-\mathrm{C}(50) 127.8(5)$} \\
\hline \multicolumn{2}{|c|}{$\mathrm{C}(45 \mathrm{~A})-\mathrm{C}(44 \mathrm{~A})-\mathrm{C}(49 \mathrm{~A}) \quad 119.5(9)$} \\
\hline \multicolumn{2}{|c|}{$\mathrm{C}(45 \mathrm{~A})-\mathrm{C}(44 \mathrm{~A})-\mathrm{C}(33) \quad 120.7(5)$} \\
\hline \multicolumn{2}{|l|}{$\mathrm{C}(49 \mathrm{~A})-\mathrm{C}(44 \mathrm{~A})-\mathrm{C}(33)$} \\
\hline \multicolumn{2}{|c|}{$C(44 A)-C(45 A)-C(46 A)$} \\
\hline \multicolumn{2}{|c|}{$\mathrm{C}(47 \mathrm{~A})-\mathrm{C}(46 \mathrm{~A})-\mathrm{C}(45 \mathrm{~A})$} \\
\hline \multicolumn{2}{|c|}{$\mathrm{C}(46 \mathrm{~A})-\mathrm{C}(47 \mathrm{~A})-\mathrm{C}(48 \mathrm{~A}) 118.9(5)$} \\
\hline $\mathrm{C}(46 \mathrm{~A})-\mathrm{C}(47 \mathrm{~A})-\mathrm{N}(4 \mathrm{~A}$ & A) $125.2(5)$ \\
\hline $\mathrm{C}(48 \mathrm{~A})-\mathrm{C}(47 \mathrm{~A})-\mathrm{N}(4 \mathrm{~A}$ & A) $115.9(4)$ \\
\hline $\mathrm{C}(49 \mathrm{~A})-\mathrm{C}(48 \mathrm{~A})-\mathrm{C}(47$ & A) $119.6(5)$ \\
\hline $\mathrm{C}(48 \mathrm{~A})-\mathrm{C}(49 \mathrm{~A})-\mathrm{C}(44$ & A) $121.1(7)$ \\
\hline $\mathrm{N}(4 \mathrm{~A})-\mathrm{C}(50 \mathrm{~A})-\mathrm{C}(51 \mathrm{~A}$ & A) $109.7(5)$ \\
\hline $\mathrm{N}(4 \mathrm{~A})-\mathrm{C}(50 \mathrm{~A})-\mathrm{C}(52 \mathrm{~A}$ & A) $110.4(6)$ \\
\hline$C(51 A)-C(50 A)-C(52$ & A) $110.7(5)$ \\
\hline$N(4 A)-C(50 A)-C(53 A$ & A) $109.9(5)$ \\
\hline$C(51 A)-C(50 A)-C(53$ & A) $106.4(6)$ \\
\hline $\mathrm{C}(52 \mathrm{~A})-\mathrm{C}(50 \mathrm{~A})-\mathrm{C}(53$ & A) $109.7(6)$ \\
\hline $\mathrm{O}(4 \mathrm{~A})-\mathrm{N}(4 \mathrm{~A})-\mathrm{C}(47 \mathrm{~A})$ & ) $117.3(4)$ \\
\hline $\mathrm{O}(4 \mathrm{~A})-\mathrm{N}(4 \mathrm{~A})-\mathrm{C}(50 \mathrm{~A})$ & $116.0(4)$ \\
\hline $\mathrm{C}(47 \mathrm{~A})-\mathrm{N}(4 \mathrm{~A})-\mathrm{C}(50 \mathrm{~A}$ & A) $126.7(4)$ \\
\hline$C(33)-C(54)-C(60)$ & $120.51(18)$ \\
\hline $\mathrm{C}(33)-\mathrm{C}(54)-\mathrm{C}(55)$ & $119.88(18)$ \\
\hline $\mathrm{C}(60)-\mathrm{C}(54)-\mathrm{C}(55)$ & $119.60(17)$ \\
\hline$C(54)-C(55)-C(64)$ & $115.80(18)$ \\
\hline $\mathrm{C}(54)-\mathrm{C}(55)-\mathrm{C}(56)$ & $110.35(18)$ \\
\hline
\end{tabular}




$\begin{array}{ll}\mathrm{C}(64)-\mathrm{C}(55)-\mathrm{C}(56) & 112.25(18) \\ \mathrm{C}(57)-\mathrm{C}(56)-\mathrm{C}(55) & 116.70(19) \\ \mathrm{C}(58)-\mathrm{C}(57)-\mathrm{C}(56) & 116.6(2) \\ \mathrm{C}(57)-\mathrm{C}(58)-\mathrm{C}(59) & 114.5(2) \\ \mathrm{C}(58)-\mathrm{C}(59)-\mathrm{C}(60) & 115.11(18) \\ \mathrm{C}(54)-\mathrm{C}(60)-\mathrm{C}(59) & 114.54(17) \\ \mathrm{C}(54)-\mathrm{C}(60)-\mathrm{C}(61) & 110.68(17) \\ \mathrm{C}(59)-\mathrm{C}(60)-\mathrm{C}(61) & 112.72(18) \\ \mathrm{C}(62)-\mathrm{C}(61)-\mathrm{C}(60) & 117.08(18) \\ \mathrm{C}(61)-\mathrm{C}(62)-\mathrm{C}(63) & 116.3(2) \\ \mathrm{C}(64)-\mathrm{C}(63)-\mathrm{C}(62) & 114.0(2) \\ \mathrm{C}(63)-\mathrm{C}(64)-\mathrm{C}(55) & 115.54(18) \\ \mathrm{O}(1)-\mathrm{N}(1)-\mathrm{C}(5) & 116.7(2) \\ \mathrm{O}(1)-\mathrm{N}(1)-\mathrm{C}(8) & 117.39(19) \\ \mathrm{C}(5)-\mathrm{N}(1)-\mathrm{C}(8) & 125.91(19) \\ \mathrm{O}(2)-\mathrm{N}(2)-\mathrm{C}(15) & 116.90(18) \\ \mathrm{O}(2)-\mathrm{N}(2)-\mathrm{C}(18) 117.00(18) \\ \mathrm{C}(15)-\mathrm{N}(2)-\mathrm{C}(18) 126.10(17) \\ \mathrm{O}(3)-\mathrm{N}(3)-\mathrm{C}(37) 116.7(2) \\ \mathrm{O}(3)-\mathrm{N}(3)-\mathrm{C}(40) 117.4(2) \\ \mathrm{C}(37)-\mathrm{N}(3)-\mathrm{C}(40) 125.7(2)\end{array}$

Symmetry transformations used to generate equivalent atoms:

Table S4. Anisotropic displacement parameters $\left(\mathrm{A}^{\wedge} 2 \times 10^{\wedge} 3\right)$ for $\mathbf{r f b i s}\left(\mathbf{1}\left(\mathbf{P h N i t}_{\mathbf{2}}\right)\right)$. The anisotropic displacement factor exponent takes the form: $-2 \mathrm{pi}^{\wedge} 2\left[\mathrm{~h}^{\wedge} 2 \mathrm{a}^{* \wedge} 2 \mathrm{U} 11+\ldots+2 \mathrm{~h} \mathrm{k} \mathrm{a*} \mathrm{b}^{*} \mathrm{U} 12\right]$

\begin{tabular}{ll}
\hline U11 & U22 U33 U23 U13 U12 \\
\hline $\mathrm{C}(1)$ & $21(1) 19(1) 20(1) 4(1) 7(1) 7(1)$ \\
$\mathrm{C}(2)$ & $21(1) 19(1) 16(1) 1(1) 2(1) 7(1)$ \\
$\mathrm{C}(3)$ & $22(1) 20(1) 19(1) 3(1) 2(1) 7(1)$ \\
$\mathrm{C}(4)$ & $21(1) 22(1) 20(1) 3(1) 2(1) 5(1)$ \\
$\mathrm{C}(5)$ & $24(1) 23(1) 19(1)-2(1)-2(1) 10(1)$ \\
$\mathrm{C}(6)$ & $32(1) 26(1) 22(1) 5(1)-1(1) 14(1)$ \\
$\mathrm{C}(7)$ & $29(1) 22(1) 22(1) 6(1) 4(1) 9(1)$ \\
$\mathrm{C}(8)$ & $20(1) 41(1) 27(1) 2(1) 2(1) 12(1)$ \\
$\mathrm{C}(9)$ & $26(1) 36(1) 40(1) 0(1) 4(1) 5(1)$ \\
$\mathrm{C}(10)$ & $26(1) 62(2) 38(1) 1(1)-4(1) 19(1)$ \\
$\mathrm{C}(11)$ & $34(1) 68(2) 31(1) 6(1) 7(1) 19(1)$ \\
$\mathrm{C}(12)$ & $21(1) 20(1) 20(1) 3(1) 4(1) 9(1)$ \\
$\mathrm{C}(13)$ & $38(1) 24(1) 28(1) 10(1) 17(1) 12(1)$ \\
$\mathrm{C}(14)$ & $39(1) 21(1) 31(1) 9(1) 15(1) 12(1)$ \\
$\mathrm{C}(15)$ & $27(1) 22(1) 21(1) 5(1) 8(1) 12(1)$ \\
$\mathrm{C}(16)$ & $26(1) 23(1) 19(1) 6(1) 7(1) 9(1)$ \\
$\mathrm{C}(17)$ & $21(1) 20(1) 21(1) 5(1) 3(1) 7(1)$ \\
$\mathrm{C}(18)$ & $26(1) 25(1) 20(1) 2(1) 8(1) 10(1)$ \\
$\mathrm{C}(19)$ & $47(2) 29(1) 28(1) 0(1) 12(1) 16(1)$ \\
$\mathrm{C}(20)$ & $31(1) 38(1) 27(1) 7(1) 2(1) 13(1)$ \\
$\mathrm{C}(21)$ & $26(1) 41(1) 35(1) 6(1) 3(1) 11(1)$ \\
$\mathrm{C}(22)$ & $20(1) 20(1) 22(1) 4(1) 6(1) 6(1)$ \\
$\mathrm{C}(23)$ & $19(1) 27(1) 29(1) 0(1) 5(1) 6(1)$ \\
$\mathrm{C}(24)$ & $23(1) 39(1) 38(1) 1(1)-1(1) 14(1)$ \\
$\mathrm{C}(25)$ & $42(2) 40(1) 40(1) 12(1)-1(1) 20(1)$ \\
$\mathrm{C}(26)$ & $36(1) 39(1) 28(1) 10(1) 2(1) 11(1)$ \\
$\mathrm{C}(27)$ & $26(1) 33(1) 25(1) 6(1) 8(1) 9(1)$ \\
$\mathrm{C}(28)$ & $20(1) 22(1) 21(1)-1(1) 4(1) 8(1)$ \\
$\mathrm{C}(29)$ & $31(1) 23(1) 27(1)-1(1) 3(1) 8(1)$
\end{tabular}




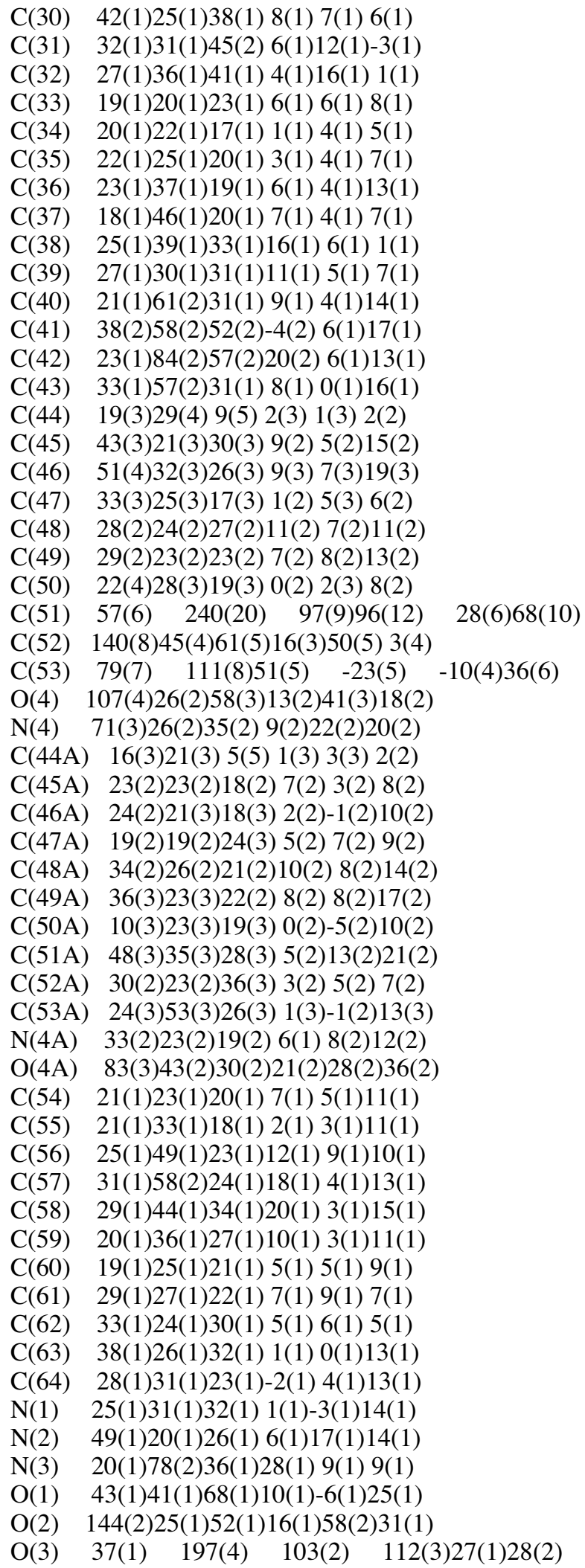

Table S5. Hydrogen coordinates $\left(\times 10^{\wedge} 4\right)$ and isotropic displacement parameters $\left(\mathrm{A}^{\wedge} 2 \times 10^{\wedge} 3\right)$ for rfbis $\left(\mathbf{1}\left(\mathrm{PhNit}_{2}\right)\right)$. 


\begin{tabular}{|c|c|c|c|c|}
\hline \multirow{2}{*}{$\begin{array}{l}\text { x y z } \\
\mathrm{H}(3 \mathrm{~A})\end{array}$} & \multicolumn{4}{|l|}{$\mathrm{U}(\mathrm{eq})$} \\
\hline & 4965 & 2999 & 3851 & 25 \\
\hline $\mathrm{H}(4 \mathrm{~A})$ & 6813 & 3122 & 4010 & 27 \\
\hline $\mathrm{H}(6 \mathrm{~A})$ & 6021 & 972 & 5162 & 32 \\
\hline $\mathrm{H}(7 \mathrm{~A})$ & 4181 & 863 & 5008 & 30 \\
\hline $\mathrm{H}(9 \mathrm{~A})$ & 8102 & 4121 & 4953 & 58 \\
\hline $\mathrm{H}(9 \mathrm{~B})$ & 9360 & 4486 & 5366 & 58 \\
\hline $\mathrm{H}(9 \mathrm{C})$ & 8390 & 3883 & 5780 & 58 \\
\hline $\mathrm{H}(10 \mathrm{~A})$ & ) 9556 & 2742 & 5679 & 68 \\
\hline $\mathrm{H}(10 \mathrm{~B})$ & 10373 & 3284 & 5133 & 68 \\
\hline $\mathrm{H}(10 \mathrm{C})$ & 9731 & 2064 & 4772 & 68 \\
\hline $\mathrm{H}(11 \mathrm{~A})$ & 8942 & 2207 & 3471 & 70 \\
\hline $\mathrm{H}(11 \mathrm{~B})$ & 9612 & 3422 & 3820 & 70 \\
\hline $\mathrm{H}(11 \mathrm{C})$ & 8305 & 3013 & 3543 & 70 \\
\hline $\mathrm{H}(13 \mathrm{~A})$ & ) 3592 & 3804 & 4539 & 35 \\
\hline $\mathrm{H}(14 \mathrm{~A})$ & 3339 & 5117 & 5644 & 35 \\
\hline $\mathrm{H}(16 \mathrm{~A})$ & 1861 & 3029 & 6758 & 27 \\
\hline $\mathrm{H}(17 \mathrm{~A})$ & 2151 & 1731 & 5658 & 25 \\
\hline $\mathrm{H}(19 \mathrm{~A})$ & 2814 & 6528 & 8430 & 55 \\
\hline H(19B) & 1720 & 6087 & 8816 & 55 \\
\hline $\mathrm{H}(19 \mathrm{C})$ & 1631 & 6365 & 7977 & 55 \\
\hline $\mathrm{H}(20 \mathrm{~A})$ & 2520 & 3884 & 7946 & 49 \\
\hline $\mathrm{H}(20 \mathrm{~B})$ & 2199 & 4562 & 8790 & 49 \\
\hline $\mathrm{H}(20 \mathrm{C})$ & 3322 & 5037 & 8449 & 49 \\
\hline $\mathrm{H}(21 \mathrm{~A})$ & 379 & 4614 & 7139 & 54 \\
\hline$H(21 B)$ & 350 & 4367 & 8000 & 54 \\
\hline $\mathrm{H}(21 \mathrm{C})$ & 654 & 3626 & 7183 & 54 \\
\hline $\mathrm{H}(23 \mathrm{~A})$ & 1198 & 1642 & 4000 & 33 \\
\hline $\mathrm{H}(24 \mathrm{~A})$ & 451 & 110 & 2384 & 43 \\
\hline $\mathrm{H}(24 \mathrm{~B})$ & 40 & 1064 & 2806 & 43 \\
\hline $\mathrm{H}(25 \mathrm{~A})$ & 1929 & 2137 & 2672 & 48 \\
\hline $\mathrm{H}(25 \mathrm{~B})$ & 994 & 1614 & 1871 & 48 \\
\hline $\mathrm{H}(26 \mathrm{~A})$ & 1724 & 219 & 1393 & 43 \\
\hline$H(26 B)$ & 2529 & 1300 & 1377 & 43 \\
\hline $\mathrm{H}(27 \mathrm{~A})$ & 3867 & 1035 & 2148 & 35 \\
\hline $\mathrm{H}(28 \mathrm{~A})$ & 3521 & 327 & 3207 & 27 \\
\hline$H(29 A)$ & 1476 & -853 & 2167 & 35 \\
\hline $\mathrm{H}(29 B)$ & 2484 & -1225 & 2324 & 35 \\
\hline $\mathrm{H}(30 \mathrm{~A})$ & 1295 & -1980 & 3052 & 44 \\
\hline $\mathrm{H}(30 \mathrm{~B})$ & 1983 & -930 & 3789 & 44 \\
\hline $\mathrm{H}(31 \mathrm{~A})$ & -301 & -1509 & 3251 & 50 \\
\hline $\mathrm{H}(32 \mathrm{~A})$ & 209 & 307 & 4445 & 47 \\
\hline $\mathrm{H}(35 \mathrm{~A})$ & ) 3679 & 8955 & 10217 & 28 \\
\hline $\mathrm{H}(36 \mathrm{~A})$ & 1789 & 8627 & 10139 & 32 \\
\hline $\mathrm{H}(38 \mathrm{~A})$ & 1460 & 6221 & 10958 & 41 \\
\hline $\mathrm{H}(39 \mathrm{~A})$ & 3347 & 6532 & 11005 & 35 \\
\hline $\mathrm{H}(41 \mathrm{~A})$ & -327 & 8808 & 10868 & 83 \\
\hline $\mathrm{H}(41 \mathrm{~B})$ & -957 & 8629 & 9974 & 83 \\
\hline $\mathrm{H}(41 \mathrm{C})$ & 358 & 8926 & 10106 & 83 \\
\hline $\mathrm{H}(42 \mathrm{~A})$ & )-1746 & 6044 & 9870 & 86 \\
\hline $\mathrm{H}(42 \mathrm{~B})$ & -2150 & 6882 & 9645 & 86 \\
\hline $\mathrm{H}(42 \mathrm{C})$ & -1799 & 7038 & 10607 & 86 \\
\hline $\mathrm{H}(43 \mathrm{~A})$ & 535 & 7463 & 9050 & 63 \\
\hline $\mathrm{H}(43 \mathrm{~B})$ & -748 & 7093 & 8699 & 63 \\
\hline $\mathrm{H}(43 \mathrm{C})$ & -242 & 6305 & 8910 & 63 \\
\hline $\mathrm{H}(45 \mathrm{~A})$ & 5094 & 7821 & 9059 & 36 \\
\hline
\end{tabular}




\begin{tabular}{|c|c|c|c|}
\hline H(46A) 5337 & 6501 & 7927 & 42 \\
\hline H(48A) 5795 & 5075 & 9570 & 30 \\
\hline $\mathrm{H}(49 \mathrm{~A}) 5514$ & 6372 & 10656 & 29 \\
\hline $\mathrm{H}(51 \mathrm{~A}) 7009$ & 3803 & 7654 & 177 \\
\hline $\mathrm{H}(51 \mathrm{~B}) 7670$ & 5005 & 8136 & 177 \\
\hline $\mathrm{H}(51 \mathrm{C}) 7819$ & 4469 & 7179 & 177 \\
\hline H(52A) 6419 & 6077 & 7028 & 133 \\
\hline $\mathrm{H}(52 \mathrm{~B}) 7474$ & 5730 & 6809 & 133 \\
\hline $\mathrm{H}(52 \mathrm{C}) 7350$ & 6280 & 7770 & 133 \\
\hline H(53A) 5107 & 4119 & 6267 & 139 \\
\hline $\mathrm{H}(53 \mathrm{~B}) 5405$ & 3265 & 6549 & 139 \\
\hline $\mathrm{H}(53 \mathrm{C}) 6212$ & 3872 & 6035 & 139 \\
\hline $\mathrm{H}(45 \mathrm{~B}) 5635$ & 6378 & 10227 & 26 \\
\hline H(46B) 5996 & 5399 & 8939 & 26 \\
\hline H(48B) 5119 & 7202 & 7822 & 30 \\
\hline H(49B) 4754 & 8140 & 9111 & 30 \\
\hline H(51D) 6768 & 4927 & 6113 & 55 \\
\hline $\mathrm{H}(51 \mathrm{E}) 5470$ & 4303 & 5921 & 55 \\
\hline$H(51 F) 6319$ & 3741 & 6050 & 55 \\
\hline $\mathrm{H}(52 \mathrm{D}) 4466$ & 3817 & 7085 & 48 \\
\hline H(52E) 5162 & 4114 & 7983 & 48 \\
\hline $\mathrm{H}(52 \mathrm{~F}) 5318$ & 3242 & & 48 \\
\hline $\mathrm{H}(53 \mathrm{D}) 7801$ & 5383 & 7483 & 56 \\
\hline $\mathrm{H}(53 \mathrm{E}) 7356$ & 4191 & 7405 & 56 \\
\hline $\mathrm{H}(53 \mathrm{~F}) 7205$ & 5051 & 8220 & 56 \\
\hline $\mathrm{H}(55 \mathrm{~A}) 4524$ & 9251 & 11904 & 30 \\
\hline $\mathrm{H}(56 \mathrm{~A}) 4526$ & 8435 & 12883 & 40 \\
\hline $\mathrm{H}(57 \mathrm{~A}) 6501$ & 8982 & 13714 & 45 \\
\hline H(58A) 6609 & 7700 & 12089 & 40 \\
\hline H(58B) 7632 & 8265 & 12826 & 40 \\
\hline H(59A) 8305 & 8995 & 11890 & 33 \\
\hline H(59B) 7794 & 9827 & 12432 & 33 \\
\hline H(60A) 6989 & 8376 & 10789 & 27 \\
\hline $\mathrm{H}(61 \mathrm{~A}) 7602$ & 9834 & 10395 & 33 \\
\hline H(62A) 8047 & 11550 & 11693 & 38 \\
\hline $\mathrm{H}(63 \mathrm{~A}) 6375$ & 11768 & 12112 & 41 \\
\hline $\mathrm{H}(63 \mathrm{~B}) 5712$ & 10733 & 11352 & 41 \\
\hline $\mathrm{H}(64 \mathrm{~A}) 5469$ & 10806 & 12831 & 35 \\
\hline H(64B) 6602 & 10572 & 12837 & 35 \\
\hline
\end{tabular}

Brown needles of notbu1 (2(PhNit $))$ were crystallized from a heptane/dichloromethane solution at $25 \mathrm{deg}$. C. A crystal of dimensions $0.44 \times 0.02 \times 0.02 \mathrm{~mm}$ was mounted on a standard Bruker SMART CCD-based X-ray diffractometer equipped with a LT-2 low temperature device and normal focus Mo-target X-ray tube $(\lambda=0.71073$ A) operated at $2000 \mathrm{~W}$ power $(50 \mathrm{kV}, 40 \mathrm{~mA})$. The X-ray intensities were measured at $158(2) \mathrm{K}$; the detector was placed at a distance $4.959 \mathrm{~cm}$ from the crystal. A total of 2242 frames were collected with a scan width of $0.3^{\circ}$ in $\omega$ and phi with an exposure time of $60 \mathrm{~s} /$ frame. The frames were integrated with the Bruker SAINT software package with a narrow frame algorithm. The integration of the data yielded a total of 12963 reflections to a maximum $2 \theta$ value of $47.92^{\circ}$ of which 4193 were independent and 2142 were greater than $2 \sigma(\mathrm{I})$. The final cell constants (Table 1) were based on the xyz centroids of 1486 reflections above $10 \sigma(\mathrm{I})$. Analysis of the data showed negligible decay during data collection; the data were processed with SADABS, no correction for absorption was necessary. The structure was solved and refined with the Bruker SHELXTL (version 5.10) software package, using the space group P1bar with $\mathrm{Z}=2$ for the formula $\mathrm{C}_{30} \mathrm{H}_{44} \mathrm{~N}_{2} \mathrm{O}_{2}$. All non-hydrogen atoms were refined anisotropically with the hydrogen atoms placed in idealized positions. Full matrix least-squares refinement based on $\mathrm{F}^{2}$ converged at $\mathrm{R} 1=$ 0.0544 and $w R 2=0.1252$ [based on $\mathrm{I}>2 \operatorname{sigma}(\mathrm{I})$ ], R1 $=0.1127$ and $\mathrm{wR} 2=0.1303$ for all data. Additional details are presented in Table 1 and are given as Supporting Information as a CIF file.

Sheldrick, G.M. SHELXTL, v. 5.10; Bruker Analytical X-ray, Madison, WI, 1997.

Sheldrick, G.M. SADABS. Program for Empirical Absorption Correction of Area Detector Data, University of Gottingen: Gottingen, Germany, 1996. 
Saint Plus, v. 6.02, Bruker Analytical X-ray, Madison, WI, 1999.

Table S6. Crystal data and structure refinement for notbu1 (2(PhNit $\left.\left.\mathbf{2}_{\mathbf{2}}\right)\right)$.

Identification code

notbu 1

Empirical formula

C30 H44 N2 O2

Formula weight

Temperature

464.67

Wavelength

$158(2) \mathrm{K}$

Crystal system, space group

$0.71073 \mathrm{~A}$

Unit cell dimensions

Triclinic, $\mathrm{P}-1$

$\mathrm{a}=6.1904(11)$ A alpha $=85.858(8) \mathrm{deg}$.

$\mathrm{b}=12.730(3) \mathrm{A} \quad$ beta $=80.307(8) \mathrm{deg}$.

$\mathrm{c}=17.528(4) \mathrm{A}$ gamma $=83.199(9) \mathrm{deg}$.

Volume

$1350.0(4) \mathrm{A}^{\wedge} 3$

Z, Calculated density

$2,1.143 \mathrm{Mg} / \mathrm{m}^{\wedge} 3$

Absorption coefficient

$0.071 \mathrm{~mm}^{\wedge}-1$

$\mathrm{F}(000)$

508

Crystal size

Theta range for data collection

$0.44 \times 0.02 \times 0.02 \mathrm{~mm}$

1.95 to $23.96 \mathrm{deg}$.

Limiting indices

Reflections collected / unique

$-6<=\mathrm{h}<=7,-14<=\mathrm{k}<=14,0<=\mathrm{l}<=20$

$12963 / 4193$ [R(int) $=0.0656]$

Completeness to theta $=$

Absorption correction

$23.96 \quad 99.6 \%$

None

Refinement method

Full-matrix least-squares on $\mathrm{F}^{\wedge} 2$

Data / restraints / parameters

Goodness-of-fit on $\mathrm{F}^{\wedge} 2$

4193 / 0 / 321

0.958

Final $\mathrm{R}$ indices [I $>2 \operatorname{sigma}(\mathrm{I})]$

$\mathrm{R} 1=0.0544, \mathrm{wR} 2=0.1127$

$\mathrm{R}$ indices (all data)

Extinction coefficient

$\mathrm{R} 1=0.1252, \mathrm{wR} 2=0.1303$

Largest diff. peak and hole

$0.027(2)$

0.195 and -0.208 e. $\mathrm{A}^{\wedge}-3$

Table S7. Atomic coordinates ( x 10^4) and equivalent isotropic displacement parameters $\left(\mathrm{A}^{\wedge} 2 \mathrm{x} 10^{\wedge} 3\right)$ for notbu1. $\left(\mathbf{2}\left(\mathbf{P h N i t}_{2}\right)\right)$

$\mathrm{U}(\mathrm{eq})$ is defined as one third of the trace of the orthogonalized

Uij tensor.

\begin{tabular}{|c|c|}
\hline $\mathrm{x} \mathrm{y} \mathrm{z}$ & $\mathrm{U}(\mathrm{eq})$ \\
\hline $\mathrm{O}(1)$ & 7482(4) 4206(2) 3830(1) 36(1) \\
\hline $\mathrm{O}(2)$ & $-2024(4) 6857(2)-671(1) 40(1)$ \\
\hline $\mathrm{N}(1)$ & $5397(4) 4279(2) 3814(1) 25(1)$ \\
\hline $\mathrm{N}(2)$ & 53(4) 6719(2) -644(1) 24(1) \\
\hline $\mathrm{C}(1)$ & $4118(5) 3426(2) 4260(2) 26(1)$ \\
\hline $\mathrm{C}(2)$ & 2091(5) 3934(3) 4766(2) 32(1) \\
\hline $\mathrm{C}(3)$ & $5626(6) 2797(3) 4769(2) 40(1)$ \\
\hline $\mathrm{C}(4)$ & $3563(6) 2691(3) 3690(2) 39(1)$ \\
\hline $\mathrm{C}(5)$ & $4485(5) 5199(2) 3423(2) 22(1)$ \\
\hline $\mathrm{C}(6)$ & $5742(5) 6054(2) 3267(2) 24(1)$ \\
\hline $\mathrm{C}(7)$ & 4952(5) 6961(2) 2878(2) 23(1) \\
\hline $\mathrm{C}(8)$ & $2924(5) 7063(2) 2630(2) 21(1)$ \\
\hline $\mathrm{C}(9)$ & $1751(5) 6179(3) 2754(2) 26(1)$ \\
\hline $\mathrm{C}(10)$ & $2470(5) 5267(2) 3161(2) 24(1)$ \\
\hline $\mathrm{C}(11)$ & $2151(5) 8036(2) 2168(2) 20(1)$ \\
\hline $\mathrm{C}(12)$ & $1711(5) 7728(2) 1410(2) 20(1)$ \\
\hline $\mathrm{C}(13)$ & $-357(5) 7996(2) 1194(2) 22(1)$ \\
\hline $\mathrm{C}(14)$ & $-848(5) 7679(2) 517(2) 23(1)$ \\
\hline $\mathrm{C}(15)$ & $725(5) 7061(2) \quad 21(2) 21(1)$ \\
\hline$C(16)$ & $2790(5) 6767(2) 235(2) 24(1)$ \\
\hline $\mathrm{C}(17)$ & $3253(5) 7089(2) 917(2) 24(1)$ \\
\hline $\mathrm{C}(18)$ & $1516(5) 6167(2)-1305(2) 23(1)$ \\
\hline
\end{tabular}


C(19) 2140(6) 5019(3)-1055(2) 43(1)

$\mathrm{C}(20) \quad 176(5) 6200(3)-1961(2) 40(1)$

$\mathrm{C}(21)$ 3549(5) 6749(3)-1598(2) 32(1)

C(22) 1897(5) 9044(3) 2408(2) 22(1)

$\mathrm{C}(23)$ 2111(5)10001(2) 1797(2) 25(1)

C(24) 3507(5) 9696(3) 1009(2) 32(1)

$\mathrm{C}(25)-66(5) 10575(3) 1607(2) 35(1)$

C(26) 3434(5)10839(2) 2053(2) 34(1)

$\mathrm{C}(27)$ 1351(5) 9264(2) 3290(2) 25(1)

$\mathrm{C}(28) \quad 320(5) 8336(3) 3790(2) 35(1)$

$\mathrm{C}(29)-464(5) 10206(3) 3448(2) 35(1)$

C(30) 3351(5) 9468(3) 3656(2) 35(1)

Table S8. Bond lengths $[\mathrm{A}]$ and angles $[\mathrm{deg}]$ for notbu1 (2(PhNit $\left.{ }_{2}\right)$ ).

\begin{tabular}{ll}
\hline $\mathrm{O}(1)-\mathrm{N}(1)$ & $1.288(3)$ \\
$\mathrm{O}(2)-\mathrm{N}(2)$ & $1.285(3)$ \\
$\mathrm{N}(1)-\mathrm{C}(5)$ & $1.421(4)$ \\
$\mathrm{N}(1)-\mathrm{C}(1)$ & $1.513(4)$ \\
$\mathrm{N}(2)-\mathrm{C}(15)$ & $1.413(4)$ \\
$\mathrm{N}(2)-\mathrm{C}(18)$ & $1.506(4)$ \\
$\mathrm{C}(1)-\mathrm{C}(2)$ & $1.515(4)$ \\
$\mathrm{C}(1)-\mathrm{C}(3)$ & $1.520(4)$ \\
$\mathrm{C}(1)-\mathrm{C}(4)$ & $1.522(4)$ \\
$\mathrm{C}(5)-\mathrm{C}(10)$ & $1.391(4)$ \\
$\mathrm{C}(5)-\mathrm{C}(6)$ & $1.396(4)$ \\
$\mathrm{C}(6)-\mathrm{C}(7)$ & $1.380(4)$ \\
$\mathrm{C}(7)-\mathrm{C}(8)$ & $1.385(4)$ \\
$\mathrm{C}(8)-\mathrm{C}(9)$ & $1.395(4)$ \\
$\mathrm{C}(8)-\mathrm{C}(11)$ & $1.504(4)$ \\
$\mathrm{C}(9)-\mathrm{C}(10)$ & $1.384(4)$ \\
$\mathrm{C}(11)-\mathrm{C}(22)$ & $1.361(4)$ \\
$\mathrm{C}(11)-\mathrm{C}(12)$ & $1.488(4)$ \\
$\mathrm{C}(12)-\mathrm{C}(13)$ & $1.393(4)$ \\
$\mathrm{C}(12)-\mathrm{C}(17)$ & $1.401(4)$ \\
$\mathrm{C}(13)-\mathrm{C}(14)$ & $1.372(4)$ \\
$\mathrm{C}(14)-\mathrm{C}(15)$ & $1.399(4)$ \\
$\mathrm{C}(15)-\mathrm{C}(16)$ & $1.394(4)$ \\
$\mathrm{C}(16)-\mathrm{C}(17)$ & $1.376(4)$ \\
$\mathrm{C}(18)-\mathrm{C}(19)$ & $1.519(4)$ \\
$\mathrm{C}(18)-\mathrm{C}(20)$ & $1.523(4)$ \\
$\mathrm{C}(18)-\mathrm{C}(21)$ & $1.532(4)$ \\
$\mathrm{C}(22)-\mathrm{C}(23)$ & $1.566(4)$ \\
$\mathrm{C}(22)-\mathrm{C}(27)$ & $1.565(4)$ \\
$\mathrm{C}(23)-\mathrm{C}(25)$ & $1.531(4)$ \\
$\mathrm{C}(23)-\mathrm{C}(24)$ & $1.550(4)$ \\
$\mathrm{C}(23)-\mathrm{C}(26)$ & $1.552(4)$ \\
$\mathrm{C}(27)-\mathrm{C}(30)$ & $1.543(4)$ \\
$\mathrm{C}(27)-\mathrm{C}(29)$ & $1.549(4)$ \\
$\mathrm{C}(27)-\mathrm{C}(28)$ & $1.553(4)$ \\
$\mathrm{O}(1)-\mathrm{N}(1)-\mathrm{C}(5)$ & $116.5(2)$ \\
$\mathrm{O}(1)-\mathrm{N}(1)-\mathrm{C}(1)$ & $117.4(2)$ \\
$\mathrm{C}(5)-\mathrm{N}(1)-\mathrm{C}(1)$ & $125.9(3)$ \\
$\mathrm{O}(2)-\mathrm{N}(2)-\mathrm{C}(15)$ & $116.6(2)$ \\
$\mathrm{O}(2)-\mathrm{N}(2)-\mathrm{C}(18)$ & $116.9(2)$ \\
$\mathrm{C}(15)-\mathrm{N}(2)-\mathrm{C}(18) 126.5(3)$ \\
$\mathrm{N}(1)-\mathrm{C}(1)-\mathrm{C}(2)$ & $109.6(3)$ \\
$\mathrm{N}(1)-\mathrm{C}(1)-\mathrm{C}(3)$ & $107.0(3)$ \\
&
\end{tabular}




$\begin{array}{ll}\mathrm{C}(2)-\mathrm{C}(1)-\mathrm{C}(3) & 109.4(3) \\ \mathrm{N}(1)-\mathrm{C}(1)-\mathrm{C}(4) & 109.1(2) \\ \mathrm{C}(2)-\mathrm{C}(1)-\mathrm{C}(4) & 112.8(3) \\ \mathrm{C}(3)-\mathrm{C}(1)-\mathrm{C}(4) & 108.8(3) \\ \mathrm{C}(10)-\mathrm{C}(5)-\mathrm{C}(6) & 119.1(3) \\ \mathrm{C}(10)-\mathrm{C}(5)-\mathrm{N}(1) & 123.3(3) \\ \mathrm{C}(6)-\mathrm{C}(5)-\mathrm{N}(1) & 117.5(3) \\ \mathrm{C}(7)-\mathrm{C}(6)-\mathrm{C}(5) & 120.0(3) \\ \mathrm{C}(6)-\mathrm{C}(7)-\mathrm{C}(8) & 122.2(3) \\ \mathrm{C}(7)-\mathrm{C}(8)-\mathrm{C}(9) & 116.8(3) \\ \mathrm{C}(7)-\mathrm{C}(8)-\mathrm{C}(11) & 121.2(3) \\ \mathrm{C}(9)-\mathrm{C}(8)-\mathrm{C}(11) & 121.6(3) \\ \mathrm{C}(10)-\mathrm{C}(9)-\mathrm{C}(8) & 122.3(3) \\ \mathrm{C}(9)-\mathrm{C}(10)-\mathrm{C}(5) & 119.5(3) \\ \mathrm{C}(22)-\mathrm{C}(11)-\mathrm{C}(12) & 125.3(3) \\ \mathrm{C}(22)-\mathrm{C}(11)-\mathrm{C}(8) 125.2(3) \\ \mathrm{C}(12)-\mathrm{C}(11)-\mathrm{C}(8) 109.6(3) \\ \mathrm{C}(13)-\mathrm{C}(12)-\mathrm{C}(17) & 116.9(3) \\ \mathrm{C}(13)-\mathrm{C}(12)-\mathrm{C}(11) & 120.4(3) \\ \mathrm{C}(17)-\mathrm{C}(12)-\mathrm{C}(11) & 122.5(3) \\ \mathrm{C}(14)-\mathrm{C}(13)-\mathrm{C}(12) & 121.8(3) \\ \mathrm{C}(13)-\mathrm{C}(14)-\mathrm{C}(15) & 120.7(3) \\ \mathrm{C}(16)-\mathrm{C}(15)-\mathrm{C}(14) & 118.4(3) \\ \mathrm{C}(16)-\mathrm{C}(15)-\mathrm{N}(2) 124.3(3) \\ \mathrm{C}(14)-\mathrm{C}(15)-\mathrm{N}(2) 117.2(3) \\ \mathrm{C}(17)-\mathrm{C}(16)-\mathrm{C}(15) & 120.2(3) \\ \mathrm{C}(16)-\mathrm{C}(17)-\mathrm{C}(12) & 122.0(3) \\ \mathrm{N}(2)-\mathrm{C}(18)-\mathrm{C}(19) 109.7(3) \\ \mathrm{N}(2)-\mathrm{C}(18)-\mathrm{C}(20) 106.9(2) \\ \mathrm{C}(19)-\mathrm{C}(18)-\mathrm{C}(20) & 109.0(3) \\ \mathrm{N}(2)-\mathrm{C}(18)-\mathrm{C}(21) 111.0(2) \\ \mathrm{C}(19)-\mathrm{C}(18)-\mathrm{C}(21) & 111.9(3) \\ \mathrm{C}(20)-\mathrm{C}(18)-\mathrm{C}(21) & 108.0(2) \\ \mathrm{C}(11)-\mathrm{C}(22)-\mathrm{C}(23) & 120.0(3) \\ \mathrm{C}(11)-\mathrm{C}(22)-\mathrm{C}(27) & 120.9(3) \\ \mathrm{C}(23)-\mathrm{C}(22)-\mathrm{C}(27) & 119.1(3) \\ \mathrm{C}(25)-\mathrm{C}(23)-\mathrm{C}(24) & 105.9(3) \\ \mathrm{C}(25)-\mathrm{C}(23)-\mathrm{C}(26) & 107.7(3) \\ \mathrm{C}(24)-\mathrm{C}(23)-\mathrm{C}(26) & 102.0(3) \\ \mathrm{C}(25)-\mathrm{C}(23)-\mathrm{C}(22) & 115.6(3) \\ \mathrm{C}(24)-\mathrm{C}(23)-\mathrm{C}(22) & 113.3(2) \\ \mathrm{C}(26)-\mathrm{C}(23)-\mathrm{C}(22) & 111.3(3) \\ \mathrm{C}(30)-\mathrm{C}(27)-\mathrm{C}(29) & 108.7(3) \\ \mathrm{C}(30)-\mathrm{C}(27)-\mathrm{C}(28) & 105.8(3) \\ \mathrm{C}(29)-\mathrm{C}(27)-\mathrm{C}(28) & 102.8(2) \\ \mathrm{C}(30)-\mathrm{C}(27)-\mathrm{C}(22) & 114.6(2) \\ \mathrm{C}(29)-\mathrm{C}(27)-\mathrm{C}(22) & 111.6(2) \\ \mathrm{C}(28)-\mathrm{C}(27)-\mathrm{C}(22) & 112.6(2) \\ & \\ \end{array}$

Symmetry transformations used to generate equivalent atoms:

Table S9. Anisotropic displacement parameters $\left(\mathrm{A}^{\wedge} 2 \times 10^{\wedge} 3\right)$ for notbu $1\left(\mathbf{2}\left(\mathbf{P h N i t}_{\mathbf{2}}\right)\right)$. The anisotropic displacement factor exponent takes the form: $-2 \mathrm{pi}^{\wedge} 2$ [ $\mathrm{h}^{\wedge} 2 \mathrm{a}^{* \wedge} 2 \mathrm{U} 11+\ldots+2 \mathrm{~h} \mathrm{k} \mathrm{a}^{*} \mathrm{~b}^{*} \mathrm{U} 12$ ]

U11 U22 U33 U23 U13 U12

$\mathrm{O}(1) \quad 27(2) 36(2) 45(2) 3(1) \quad-14(1) 1(1)$

$\mathrm{O}(2) \quad 18(2) 66(2) 39(1) \quad-18(1)-9(1) 2(1)$ 
$\begin{array}{ll}\mathrm{N}(1) & 25(2) 26(2) 24(2)-1(1)-9(1) 0(1) \\ \mathrm{N}(2) & 18(2) 30(2) 25(2)-4(1)-4(1)-1(1) \\ \mathrm{C}(1) & 30(2) 23(2) 23(2) 2(2)-3(2)-3(2) \\ \mathrm{C}(2) & 33(2) 39(2) 24(2) 4(2)-4(2)-2(2) \\ \mathrm{C}(3) & 42(2) 36(2) 38(2) 11(2)-6(2) 3(2) \\ \mathrm{C}(4) & 55(3) 23(2) 39(2) 3(2)-7(2)-10(2) \\ \mathrm{C}(5) & 26(2) 22(2) 16(2)-2(2)-4(2) 2(2) \\ \mathrm{C}(6) & 22(2) 29(2) 23(2)-2(2)-7(2)-3(2) \\ \mathrm{C}(7) & 24(2) 23(2) 23(2)-2(2)-2(2)-5(2) \\ \mathrm{C}(8) & 22(2) 20(2) 21(2)-4(2)-4(2) 1(2) \\ \mathrm{C}(9) & 26(2) 29(2) 27(2)-7(2)-7(2)-5(2) \\ \mathrm{C}(10) & 25(2) 24(2) 24(2)-1(2)-7(2)-6(2) \\ \mathrm{C}(11) & 13(2) 25(2) 22(2) 1(2)-2(1)-3(2) \\ \mathrm{C}(12) & 24(2) 17(2) 19(2) 0(1)-4(2)-1(2) \\ \mathrm{C}(13) & 19(2) 22(2) 24(2) 3(2) 0(2)-2(2) \\ \mathrm{C}(14) & 19(2) 23(2) 29(2) 1(2)-6(2)-3(2) \\ \mathrm{C}(15) & 20(2) 21(2) 20(2) 1(2)-3(2)-3(2) \\ \mathrm{C}(16) & 25(2) 24(2) 24(2)-3(2)-6(2) 3(2) \\ \mathrm{C}(17) & 18(2) 28(2) 28(2) 3(2)-7(2)-2(2) \\ \mathrm{C}(18) & 23(2) 22(2) 24(2)-5(2)-3(2) 0(2) \\ \mathrm{C}(19) & 49(3) 27(2) 47(2)-1(2) 1(2) 1(2) \\ \mathrm{C}(20) & 27(2) 63(3) 32(2)-21(2)-3(2) 0(2) \\ \mathrm{C}(21) & 29(2) 37(2) 28(2) 1(2) 0(2)-6(2) \\ \mathrm{C}(22) & 12(2) 26(2) 27(2) 0(2)-3(1)-3(2) \\ \mathrm{C}(23) & 25(2) 20(2) 29(2) 0(2)-4(2)-1(2) \\ \mathrm{C}(24) & 35(2) 29(2) 32(2) 4(2)-3(2)-9(2) \\ \mathrm{C}(25) & 33(2) 32(2) 39(2) 6(2)-10(2)-1(2) \\ \mathrm{C}(26) & 33(2) 24(2) 45(2) 2(2)-6(2)-7(2) \\ \mathrm{C}(27) & 24(2) 26(2) 26(2)-5(2)-1(2)-4(2) \\ \mathrm{C}(28) & 38(2) 36(2) 27(2)-6(2) 4(2)-2(2) \\ \mathrm{C}(29) & 32(2) 35(2) 35(2)-9(2) 1(2) 0(2) \\ \mathrm{C}(30) & 39(2) 34(2) 32(2)-7(2)-11(2) 1(2) \\ & \end{array}$

Table S10. Hydrogen coordinates ( x 10^4) and isotropic displacement parameters $\left(\mathrm{A}^{\wedge} 2 \times 10^{\wedge} 3\right)$ for notbu1 $\left(\mathbf{2}\left(\mathbf{P h N i t}_{2}\right)\right)$.

\begin{tabular}{lcccc}
\hline x y z & U(eq) & & & \\
& & & & \\
\hline H(2A) & 2513 & 4481 & 5060 & $45(2)$ \\
H(2B) & 1422 & 3393 & 5126 & $45(2)$ \\
H(2C) & 1026 & 4256 & 4439 & $45(2)$ \\
H(3A) & 6929 & 2461 & 4442 & $45(2)$ \\
H(3B) & 4835 & 2251 & 5083 & $45(2)$ \\
H(3C) & 6086 & 3274 & 5111 & $45(2)$ \\
H(4A) & 2637 & 3095 & 3345 & $45(2)$ \\
H(4B) & 2768 & 2126 & 3977 & $45(2)$ \\
H(4C) & 4928 & 2379 & 3383 & $45(2)$ \\
H(6) & 7140 & 6011 & 3429 & $45(2)$ \\
H(7) & 5828 & 7535 & 2776 & $45(2)$ \\
H(9) & 412 & 6203 & 2553 & $45(2)$ \\
H(10) & 1594 & 4693 & 3261 & $45(2)$ \\
H(13) & -1458 & 8409 & 1524 & $45(2)$ \\
H(14) & -2271 & 7881 & 385 & $45(2)$ \\
H(16) & 3881 & 6342 & -91 & $45(2)$ \\
H(17) & 4661 & 6871 & 1057 & $45(2)$ \\
H(19A) 2952 & 4991 & -618 & $45(2)$ \\
H(19B) 3071 & 4659 & -1489 & $45(2)$ \\
H(19C) & 801 & 4665 & -896 & $45(2)$
\end{tabular}




\begin{tabular}{|c|c|c|c|}
\hline & & & \\
\hline & 5903 & & \\
\hline & & & \\
\hline & 7498 & & \\
\hline & & & \\
\hline & 66 & & \\
\hline & 9279 & 73 & \\
\hline & & & \\
\hline & 103 & & \\
\hline & & & \\
\hline B) 238 & 111 & & \\
\hline & & & \\
\hline & & & \\
\hline & & & \\
\hline & & 25 & \\
\hline & & 38 & \\
\hline & 81 & 354 & \\
\hline ) -504 & & & \\
\hline & & 40 & \\
\hline$[(29 B)-1795$ & 10057 & 3260 & 45( \\
\hline & 10852 & 3178 & \\
\hline ( 304$) 2005$ & & 4212 & \\
\hline & 10107 & 3407 & \\
\hline & & & \\
\hline
\end{tabular}

\section{Structure Determination.}

Brown plates of flat $2\left(\mathbf{6}\left(\mathbf{N i t}_{2}\right)\right)$ were crystallized from dichloromethane/pentane at $23 \mathrm{deg}$. C. A crystal of dimensions $0.24 \times 0.12 \times 0.06 \mathrm{~mm}$ was mounted on a standard Bruker SMART CCD-based X-ray diffractometer equipped with a LT-2 low temperature device and normal focus Mo-target X-ray tube $(\lambda=0.71073 \mathrm{~A})$ operated at $2000 \mathrm{~W}$ power $(50 \mathrm{kV}, 40 \mathrm{~mA})$. The X-ray intensities were measured at $158(2) \mathrm{K}$; the detector was placed at a distance $5.058 \mathrm{~cm}$ from the crystal. A total of 2862 frames were collected with a scan width of $0.3^{\circ}$ in $\omega$ and phi with an exposure time of $75 \mathrm{~s} /$ frame. The frames were integrated with the Bruker SAINT software package with a narrow frame algorithm. The integration of the data yielded a total of 28025 reflections to a maximum $2 \theta$ value of $52.92^{\circ}$ of which 3524 were independent and 2083 were greater than $2 \sigma(\mathrm{I})$. The final cell constants (Table 1) were based on the xyz centroids of 910 reflections above $10 \sigma(\mathrm{I})$. Analysis of the data showed negligible decay during data collection. The data were processed with SADABS, no correction for absorption was necessary. The structure was solved and refined with the Bruker SHELXTL (version 5.10) software package using the space group P2/c with $\mathrm{Z}=4$ for the formula $\mathrm{C}_{28} \mathrm{H}_{36} \mathrm{~N}_{2} \mathrm{O}_{2}$. All non-hydrogen atoms were refined anisotropically with the hydrogen atoms placed in idealized positions. Full matrix least-squares refinement based on $\mathrm{F}^{2}$ converged at $\mathrm{R} 1=0.0584$ and wR2 $=0.1330$ [based on $\mathrm{I}>2 \operatorname{sigma}(\mathrm{I})], \mathrm{R} 1=0.1185$ and $\mathrm{wR} 2=0.1642$ for all data. Additional details are presented in Table 1 and are given as Supporting Information in a CIF file.

Sheldrick, G.M. SHELXTL, v. 5.10; Bruker Analytical X-ray, Madison, WI, 1997.

Sheldrick, G.M. SADABS. Program for Empirical Absorption Correction of Area Detector Data, University of Gottingen: Gottingen, Germany, 1996.

Saint Plus, v. 6.02, Bruker Analytical X-ray, Madison, WI, 1999.

Table S11. Crystal data and structure refinement for flat2 $\left(\mathbf{6}\left(\mathbf{N i t}_{2}\right)\right)$.

Identification code

Empirical formula

Formula weight

Temperature

Wavelength

Crystal system, space group

Unit cell dimensions

Volume

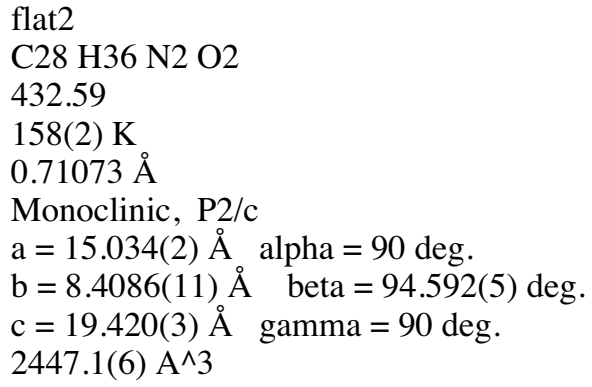

flat2

C28 H36 N2 O2

432.59

158(2) K

$0.71073 \AA$

Monoclinic, P2/c

$\mathrm{a}=15.034(2) \AA$ alpha $=90 \mathrm{deg}$.

$\mathrm{b}=8.4086(11) \AA \quad$ beta $=94.592(5) \mathrm{deg}$.

$\mathrm{c}=19.420(3) \AA$ gamma $=90 \mathrm{deg}$.

2447.1(6) $\mathrm{A}^{\wedge} 3$ 
Z, Calculated density

Absorption coefficient

$\mathrm{F}(000)$

Crystal size

Theta range for data collection

Limiting indices

Reflections collected / unique

Completeness to theta $=23.25$

Absorption correction

Refinement method

Data / restraints / parameters

Goodness-of-fit on $\mathrm{F}^{\wedge} 2$

Final $\mathrm{R}$ indices [I $>2 \operatorname{sigma}(\mathrm{I})]$

$\mathrm{R}$ indices (all data)

Extinction coefficient

Largest diff. peak and hole

Table S12. Atomic coordinates ( x 10^4) and equivalent isotropic displacement parameters $\left(\mathrm{A}^{\wedge} 2 \times 10^{\wedge} 3\right)$ for flat 2 $\left(\mathbf{6}\left(\mathrm{Nit}_{2}\right)\right)$.

$\mathrm{U}(\mathrm{eq})$ is defined as one third of the trace of the orthogonalized Uij tensor.

\begin{tabular}{lcccc}
\hline $\mathrm{x}$ & $\mathrm{y}$ & $\mathrm{U}(\mathrm{eq})$ & & \\
\hline $\mathrm{O}(1)$ & $5551(1)$ & $5053(3)$ & $5579(1)$ & $40(1)$ \\
$\mathrm{O}(2)$ & $666(2)$ & $14226(3)$ & $8479(1)$ & $51(1)$ \\
$\mathrm{N}(1)$ & $4715(2)$ & $4640(3)$ & $5631(1)$ & $31(1)$ \\
$\mathrm{N}(2)$ & $1480(2)$ & $14381(3)$ & $8320(2)$ & $34(1)$ \\
$\mathrm{C}(1)$ & $4549(2)$ & $2926(4)$ & $5808(2)$ & $34(1)$ \\
$\mathrm{C}(2)$ & $3597(2)$ & $2475(5)$ & $5558(2)$ & $54(1)$ \\
$\mathrm{C}(3)$ & $5200(3)$ & $1889(4)$ & $5456(2)$ & $45(1)$ \\
$\mathrm{C}(4)$ & $4699(3)$ & $2747(5)$ & $6591(2)$ & $44(1)$ \\
$\mathrm{C}(5)$ & $4180(2)$ & $5865(4)$ & $5917(2)$ & $29(1)$ \\
$\mathrm{C}(6)$ & $4531(2)$ & $6729(4)$ & $6486(2)$ & $32(1)$ \\
$\mathrm{C}(7)$ & $4041(2)$ & $7925(4)$ & $6762(2)$ & $30(1)$ \\
$\mathrm{C}(8)$ & $3188(2)$ & $8266(4)$ & $6458(2)$ & $27(1)$ \\
$\mathrm{C}(9)$ & $2527(2)$ & $9444(4)$ & $6618(2)$ & $27(1)$ \\
$\mathrm{C}(10)$ & $2410(2)$ & $10695(4)$ & $7115(2)$ & $27(1)$ \\
$\mathrm{C}(11)$ & $2933(2)$ & $11211(4)$ & $7700(2)$ & $30(1)$ \\
$\mathrm{C}(12)$ & $2629(2)$ & $12442(4)$ & $8088(2)$ & $32(1)$ \\
$\mathrm{C}(13)$ & $1810(2)$ & $13163(4)$ & $7900(2)$ & $27(1)$ \\
$\mathrm{C}(14)$ & $1273(2)$ & $12644(4)$ & $7320(2)$ & $28(1)$ \\
$\mathrm{C}(15)$ & $1577(2)$ & $11422(4)$ & $6934(2)$ & $27(1)$ \\
$\mathrm{C}(16)$ & $1119(2)$ & $10625(4)$ & $6281(2)$ & $27(1)$ \\
$\mathrm{C}(17)$ & $1803(2)$ & $9355(4)$ & $6146(2)$ & $28(1)$ \\
$\mathrm{C}(18)$ & $1912(2)$ & $8051(4)$ & $5621(2)$ & $28(1)$ \\
$\mathrm{C}(19)$ & $2840(2)$ & $7400(4)$ & $5876(2)$ & $26(1)$ \\
$\mathrm{C}(20)$ & $3324(2)$ & $6214(4)$ & $5603(2)$ & $28(1)$ \\
$\mathrm{C}(21)$ & $972(2)$ & $11836(4)$ & $5691(2)$ & $34(1)$ \\
$\mathrm{C}(22)$ & $214(2)$ & $9918(4)$ & $6452(2)$ & $32(1)$ \\
$\mathrm{C}(23)$ & $1936(2)$ & $8672(4)$ & $4881(2)$ & $38(1)$ \\
$\mathrm{C}(24)$ & $1199(2)$ & $6736(4)$ & $5641(2)$ & $35(1)$ \\
$\mathrm{C}(25)$ & $1832(2)$ & $16056(4)$ & $8351(2)$ & $31(1)$ \\
$\mathrm{C}(26)$ & $1288(3)$ & $16992(5)$ & $7799(2)$ & $50(1)$ \\
$\mathrm{C}(27)$ & $1696(3)$ & $16713(5)$ & $9062(2)$ & $46(1)$ \\
$\mathrm{C}(28)$ & $2815(2)$ & $16122(5)$ & $8210(2)$ & $48(1)$ \\
& & & & \\
\hline
\end{tabular}

Table S13. Bond lengths [A] and angles [deg] for flat2 $\left(\mathbf{6}\left(\mathbf{N i t}_{2}\right)\right)$.

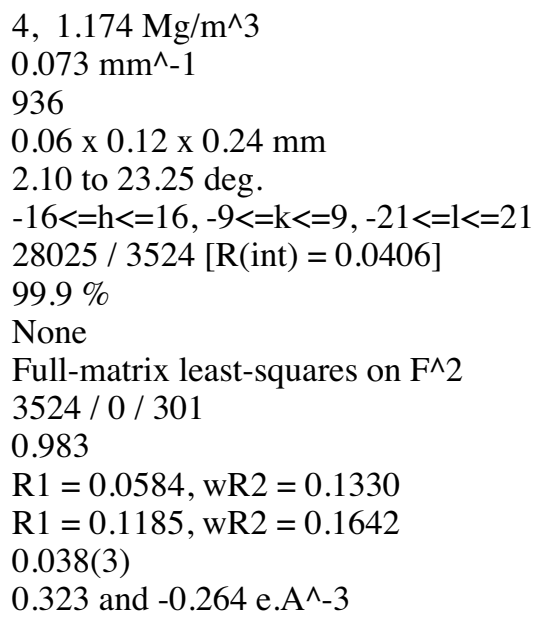




\begin{tabular}{|c|c|}
\hline $\mathrm{O}(1)-\mathrm{N}(1)$ & $1.316(3)$ \\
\hline $\mathrm{O}(2)-\mathrm{N}(2)$ & $1.292(3)$ \\
\hline $\mathrm{N}(1)-\mathrm{C}(5)$ & $1.445(4)$ \\
\hline $\mathrm{N}(1)-\mathrm{C}(1)$ & $1.507(4)$ \\
\hline $\mathrm{N}(2)-\mathrm{C}(13)$ & $1.423(4)$ \\
\hline $\mathrm{N}(2)-\mathrm{C}(25)$ & $1.504(4)$ \\
\hline $\mathrm{C}(1)-\mathrm{C}(3)$ & $1.515(5)$ \\
\hline $\mathrm{C}(1)-\mathrm{C}(2)$ & $1.522(5)$ \\
\hline$C(1)-C(4)$ & $1.527(5)$ \\
\hline$C(5)-C(6)$ & $1.391(5)$ \\
\hline$C(5)-C(20)$ & $1.409(4)$ \\
\hline$C(6)-C(7)$ & $1.380(5)$ \\
\hline $\mathrm{C}(7)-\mathrm{C}(8)$ & $1.399(4)$ \\
\hline $\mathrm{C}(8)-\mathrm{C}(19)$ & $1.410(4)$ \\
\hline $\mathrm{C}(8)-\mathrm{C}(9)$ & $1.454(4)$ \\
\hline C(9)-C(17) & $1.368(4)$ \\
\hline C(9)-C(10) & $1.447(5)$ \\
\hline$C(10)-C(11)$ & $1.399(4)$ \\
\hline C(10)-C(15) & $1.413(4)$ \\
\hline$C(11)-C(12)$ & $1.380(5)$ \\
\hline$C(12)-C(13)$ & $1.395(4)$ \\
\hline C(13)-C(14) & $1.402(4)$ \\
\hline C(14)-C(15) & $1.372(4)$ \\
\hline$C(15)-C(16)$ & $1.548(4)$ \\
\hline$C(16)-C(17)$ & $1.520(5)$ \\
\hline$C(16)-C(21)$ & $1.534(4)$ \\
\hline$C(16)-C(22)$ & $1.545(4)$ \\
\hline $\mathrm{C}(17)-\mathrm{C}(18)$ & $1.515(5)$ \\
\hline $\mathrm{C}(18)-\mathrm{C}(23)$ & $1.531(5)$ \\
\hline C(18)-C(19) & $1.542(4)$ \\
\hline $\mathrm{C}(18)-\mathrm{C}(24)$ & $1.544(5)$ \\
\hline C(19)-C(20) & $1.367(4)$ \\
\hline$C(25)-C(26)$ & $1.516(5)$ \\
\hline$C(25)-C(27)$ & $1.516(5)$ \\
\hline $\mathrm{C}(25)-\mathrm{C}(28)$ & $1.525(5)$ \\
\hline $\mathrm{O}(1)-\mathrm{N}(1)-\mathrm{C}(5)$ & $114.0(3)$ \\
\hline $\mathrm{O}(1)-\mathrm{N}(1)-\mathrm{C}(1)$ & $116.5(2)$ \\
\hline $\mathrm{C}(5)-\mathrm{N}(1)-\mathrm{C}(1)$ & $119.1(3)$ \\
\hline $\mathrm{O}(2)-\mathrm{N}(2)-\mathrm{C}(13)$ & $116.3(3)$ \\
\hline $\mathrm{O}(2)-\mathrm{N}(2)-\mathrm{C}(25)$ & $115.0(3)$ \\
\hline $\mathrm{C}(13)-\mathrm{N}(2)-\mathrm{C}(25)$ & $124.0(3)$ \\
\hline $\mathrm{N}(1)-\mathrm{C}(1)-\mathrm{C}(3)$ & $108.9(3)$ \\
\hline $\mathrm{N}(1)-\mathrm{C}(1)-\mathrm{C}(2)$ & $109.6(3)$ \\
\hline $\mathrm{C}(3)-\mathrm{C}(1)-\mathrm{C}(2)$ & $109.8(3)$ \\
\hline $\mathrm{N}(1)-\mathrm{C}(1)-\mathrm{C}(4)$ & $107.9(3)$ \\
\hline $\mathrm{C}(3)-\mathrm{C}(1)-\mathrm{C}(4)$ & $110.1(3)$ \\
\hline $\mathrm{C}(2)-\mathrm{C}(1)-\mathrm{C}(4)$ & $110.6(3)$ \\
\hline$C(6)-C(5)-C(20)$ & $120.7(3)$ \\
\hline $\mathrm{C}(6)-\mathrm{C}(5)-\mathrm{N}(1)$ & $119.3(3)$ \\
\hline $\mathrm{C}(20)-\mathrm{C}(5)-\mathrm{N}(1)$ & $120.0(3)$ \\
\hline$C(7)-C(6)-C(5)$ & $120.6(3)$ \\
\hline$C(6)-C(7)-C(8)$ & $119.1(3)$ \\
\hline$C(7)-C(8)-C(19)$ & 120.1(3) \\
\hline $\mathrm{C}(7)-\mathrm{C}(8)-\mathrm{C}(9)$ & $132.1(3)$ \\
\hline C(19)-C(8)-C(9) & $107.9(3)$ \\
\hline $\mathrm{C}(17)-\mathrm{C}(9)-\mathrm{C}(10)$ & $110.7(3)$ \\
\hline $\mathrm{C}(17)-\mathrm{C}(9)-\mathrm{C}(8)$ & $109.9(3)$ \\
\hline $\mathrm{C}(10)-\mathrm{C}(9)-\mathrm{C}(8)$ & $139.3(3)$ \\
\hline
\end{tabular}




$\begin{array}{lc}\mathrm{C}(11)-\mathrm{C}(10)-\mathrm{C}(15) & 119.8(3) \\ \mathrm{C}(11)-\mathrm{C}(10)-\mathrm{C}(9) & 132.7(3) \\ \mathrm{C}(15)-\mathrm{C}(10)-\mathrm{C}(9) & 107.5(3) \\ \mathrm{C}(12)-\mathrm{C}(11)-\mathrm{C}(10) & 119.2(3) \\ \mathrm{C}(11)-\mathrm{C}(12)-\mathrm{C}(13) & 120.5(3) \\ \mathrm{C}(12)-\mathrm{C}(13)-\mathrm{C}(14) & 121.0(3) \\ \mathrm{C}(12)-\mathrm{C}(13)-\mathrm{N}(2) & 120.2(3) \\ \mathrm{C}(14)-\mathrm{C}(13)-\mathrm{N}(2) & 118.7(3) \\ \mathrm{C}(15)-\mathrm{C}(14)-\mathrm{C}(13) & 118.4(3) \\ \mathrm{C}(14)-\mathrm{C}(15)-\mathrm{C}(10) & 121.1(3) \\ \mathrm{C}(14)-\mathrm{C}(15)-\mathrm{C}(16) & 128.8(3) \\ \mathrm{C}(10)-\mathrm{C}(15)-\mathrm{C}(16) & 110.1(3) \\ \mathrm{C}(17)-\mathrm{C}(16)-\mathrm{C}(21) & 113.4(3) \\ \mathrm{C}(17)-\mathrm{C}(16)-\mathrm{C}(22) & 112.7(3) \\ \mathrm{C}(21)-\mathrm{C}(16)-\mathrm{C}(22) & 109.8(3) \\ \mathrm{C}(17)-\mathrm{C}(16)-\mathrm{C}(15) & 100.5(3) \\ \mathrm{C}(21)-\mathrm{C}(16)-\mathrm{C}(15) & 110.6(3) \\ \mathrm{C}(22)-\mathrm{C}(16)-\mathrm{C}(15) & 109.4(3) \\ \mathrm{C}(9)-\mathrm{C}(17)-\mathrm{C}(18) & 111.5(3) \\ \mathrm{C}(9)-\mathrm{C}(17)-\mathrm{C}(16) & 111.1(3) \\ \mathrm{C}(18)-\mathrm{C}(17)-\mathrm{C}(16) & 137.4(3) \\ \mathrm{C}(17)-\mathrm{C}(18)-\mathrm{C}(23) & 113.4(3) \\ \mathrm{C}(17)-\mathrm{C}(18)-\mathrm{C}(19) & 100.7(3) \\ \mathrm{C}(23)-\mathrm{C}(18)-\mathrm{C}(19) & 109.5(3) \\ \mathrm{C}(17)-\mathrm{C}(18)-\mathrm{C}(24) & 112.8(3) \\ \mathrm{C}(23)-\mathrm{C}(18)-\mathrm{C}(24) & 109.6(3) \\ \mathrm{C}(19)-\mathrm{C}(18)-\mathrm{C}(24) & 110.5(3) \\ \mathrm{C}(20)-\mathrm{C}(19)-\mathrm{C}(8) & 120.9(3) \\ \mathrm{C}(20)-\mathrm{C}(19)-\mathrm{C}(18) & 129.1(3) \\ \mathrm{C}(8)-\mathrm{C}(19)-\mathrm{C}(18) & 110.0(3) \\ \mathrm{C}(19)-\mathrm{C}(20)-\mathrm{C}(5) & 118.7(3) \\ \mathrm{N}(2)-\mathrm{C}(25)-\mathrm{C}(26) & 106.8(3) \\ \mathrm{N}(2)-\mathrm{C}(25)-\mathrm{C}(27) & 107.8(3) \\ \mathrm{C}(26)-\mathrm{C}(25)-\mathrm{C}(27) & 110.6(3) \\ \mathrm{N}(2)-\mathrm{C}(25)-\mathrm{C}(28) & 111.7(3) \\ \mathrm{C}(26)-\mathrm{C}(25)-\mathrm{C}(28) & 109.2(3) \\ \mathrm{C}(27)-\mathrm{C}(25)-\mathrm{C}(28) & 110.7(3) \\ & \end{array}$

Symmetry transformations used to generate equivalent atoms:

Table S14. Anisotropic displacement parameters $\left(\mathrm{A}^{\wedge} 2 \times 10^{\wedge} 3\right)$ for flat2. $\left(\mathbf{6}\left(\mathbf{N i t}_{2}\right)\right)$ The anisotropic displacement factor exponent takes the form: $-2 \mathrm{pi}^{\wedge} 2\left[\mathrm{~h}^{\wedge} 2 \mathrm{a}^{* \wedge} 2 \mathrm{U} 11+\ldots+2 \mathrm{~h} \mathrm{k} \mathrm{a} \mathrm{b}^{*} \mathrm{U} 12\right.$ ]

\begin{tabular}{lcccccl}
\hline $\mathrm{U} 11$ & $\mathrm{U} 22$ & $\mathrm{U} 33$ & $\mathrm{U} 23$ & $\mathrm{U} 13$ & $\mathrm{U} 12$ \\
\hline $\mathrm{O}(1)$ & $14(1)$ & $47(2)$ & $57(2)$ & $-7(1)$ & $-4(1)$ & $2(1)$ \\
$\mathrm{O}(2)$ & $22(2)$ & $63(2)$ & $71(2)$ & $-21(2)$ & $10(1)$ & $-7(1)$ \\
$\mathrm{N}(1)$ & $16(2)$ & $39(2)$ & $35(2)$ & $-2(1)$ & $-4(1)$ & $4(1)$ \\
$\mathrm{N}(2)$ & $13(2)$ & $44(2)$ & $44(2)$ & $-5(2)$ & $-1(1)$ & $-2(1)$ \\
$\mathrm{C}(1)$ & $18(2)$ & $34(2)$ & $49(2)$ & $2(2)$ & $-9(2)$ & $-3(2)$ \\
$\mathrm{C}(2)$ & $36(2)$ & $45(3)$ & $77(3)$ & $11(2)$ & $-19(2)$ & $-14(2)$ \\
$\mathrm{C}(3)$ & $47(3)$ & $36(2)$ & $52(3)$ & $-7(2)$ & $-6(2)$ & $6(2)$ \\
$\mathrm{C}(4)$ & $38(2)$ & $44(2)$ & $50(3)$ & $4(2)$ & $-1(2)$ & $5(2)$ \\
$\mathrm{C}(5)$ & $17(2)$ & $34(2)$ & $36(2)$ & $-4(2)$ & $-3(2)$ & $2(2)$ \\
$\mathrm{C}(6)$ & $15(2)$ & $42(2)$ & $38(2)$ & $-4(2)$ & $-9(2)$ & $4(2)$ \\
$\mathrm{C}(7)$ & $19(2)$ & $36(2)$ & $34(2)$ & $-3(2)$ & $-9(2)$ & $3(2)$ \\
$\mathrm{C}(8)$ & $14(2)$ & $32(2)$ & $34(2)$ & $0(2)$ & $-5(2)$ & $3(2)$ \\
$\mathrm{C}(9)$ & $15(2)$ & $36(2)$ & $29(2)$ & $0(2)$ & $-5(2)$ & $-1(2)$
\end{tabular}




$\begin{array}{llllrrr}\mathrm{C}(10) & 14(2) & 34(2) & 31(2) & 0(2) & -4(2) & 2(2) \\ \mathrm{C}(11) & 15(2) & 41(2) & 33(2) & -2(2) & -9(2) & 1(2) \\ \mathrm{C}(12) & 19(2) & 42(2) & 31(2) & -3(2) & -9(2) & 0(2) \\ \mathrm{C}(13) & 18(2) & 33(2) & 31(2) & -7(2) & 0(2) & 0(2) \\ \mathrm{C}(14) & 17(2) & 34(2) & 33(2) & -1(2) & -3(2) & 1(2) \\ \mathrm{C}(15) & 19(2) & 30(2) & 32(2) & 6(2) & -3(2) & -4(2) \\ \mathrm{C}(16) & 15(2) & 33(2) & 33(2) & -2(2) & -4(2) & -1(2) \\ \mathrm{C}(17) & 16(2) & 36(2) & 31(2) & 1(2) & -4(2) & -1(2) \\ \mathrm{C}(18) & 13(2) & 38(2) & 30(2) & 0(2) & -9(2) & 5(2) \\ \mathrm{C}(19) & 13(2) & 31(2) & 34(2) & -1(2) & -5(2) & -1(2) \\ \mathrm{C}(20) & 20(2) & 33(2) & 30(2) & -5(2) & -7(2) & -1(2) \\ \mathrm{C}(21) & 23(2) & 41(2) & 36(2) & 4(2) & -5(2) & 2(2) \\ \mathrm{C}(22) & 14(2) & 41(2) & 39(2) & -1(2) & -5(2) & -1(2) \\ \mathrm{C}(23) & 28(2) & 46(2) & 38(2) & -4(2) & -9(2) & 10(2) \\ \mathrm{C}(24) & 19(2) & 43(2) & 42(2) & -8(2) & -5(2) & 0(2) \\ \mathrm{C}(25) & 26(2) & 29(2) & 37(2) & -1(2) & -3(2) & 0(2) \\ \mathrm{C}(26) & 42(3) & 57(3) & 48(3) & 8(2) & -10(2) & 7(2) \\ \mathrm{C}(27) & 45(3) & 47(3) & 43(2) & -8(2) & -6(2) & 1(2) \\ \mathrm{C}(28) & 25(2) & 42(2) & 75(3) & 2(2) & 2(2) & -4(2)\end{array}$

Table S15. Hydrogen coordinates ( x 10^4) and isotropic displacement parameters $\left(\mathrm{A}^{\wedge} 2 \times 10^{\wedge} 3\right)$ for flat2 $\left(\mathbf{6}\left(\mathbf{N i t}_{2}\right)\right)$.

\begin{tabular}{lcccc}
\hline X & \multicolumn{5}{c}{$\mathrm{z}$} & \multicolumn{2}{c}{ eq $)$} & \\
\hline $\mathrm{H}(2 \mathrm{~A})$ & 3178 & 3131 & 5796 & $51(2)$ \\
$\mathrm{H}(2 \mathrm{~B})$ & 3496 & 1351 & 5660 & $51(2)$ \\
$\mathrm{H}(2 \mathrm{C})$ & 3503 & 2653 & 5059 & $51(2)$ \\
$\mathrm{H}(3 \mathrm{~A})$ & 5099 & 2014 & 4954 & $51(2)$ \\
$\mathrm{H}(3 \mathrm{~B})$ & 5110 & 774 & 5580 & $51(2)$ \\
$\mathrm{H}(3 \mathrm{C})$ & 5812 & 2207 & 5606 & $51(2)$ \\
$\mathrm{H}(4 \mathrm{~A})$ & 5293 & 3148 & 6748 & $51(2)$ \\
$\mathrm{H}(4 \mathrm{~B})$ & 4654 & 1622 & 6716 & $51(2)$ \\
$\mathrm{H}(4 \mathrm{C})$ & 4245 & 3357 & 6813 & $51(2)$ \\
$\mathrm{H}(6)$ & 5113 & 6493 & 6686 & $51(2)$ \\
$\mathrm{H}(7)$ & 4281 & 8509 & 7153 & $51(2)$ \\
$\mathrm{H}(11)$ & 3491 & 10720 & 7829 & $51(2)$ \\
$\mathrm{H}(12)$ & 2979 & 12800 & 8487 & $51(2)$ \\
$\mathrm{H}(14)$ & 711 & 13128 & 7197 & $51(2)$ \\
$\mathrm{H}(20)$ & 3089 & 5635 & 5210 & $51(2)$ \\
$\mathrm{H}(21 \mathrm{~A})$ & 1545 & 12305 & 5595 & $51(2)$ \\
$\mathrm{H}(21 \mathrm{~B})$ & 569 & 12677 & 5827 & $51(2)$ \\
$\mathrm{H}(21 \mathrm{C})$ & 707 & 11301 & 5276 & $51(2)$ \\
$\mathrm{H}(22 \mathrm{~A})$ & -60 & 9354 & 6046 & $51(2)$ \\
$\mathrm{H}(22 \mathrm{~B})$ & -183 & 10777 & 6578 & $51(2)$ \\
$\mathrm{H}(22 \mathrm{C})$ & 309 & 9174 & 6839 & $51(2)$ \\
$\mathrm{H}(23 \mathrm{~A})$ & 1354 & 9130 & 4727 & $51(2)$ \\
$\mathrm{H}(23 \mathrm{~B})$ & 2070 & 7792 & 4575 & $51(2)$ \\
$\mathrm{H}(23 \mathrm{C})$ & 2398 & 9490 & 4868 & $51(2)$ \\
$\mathrm{H}(24 \mathrm{~A})$ & 1186 & 6340 & 6114 & $51(2)$ \\
$\mathrm{H}(24 \mathrm{~B})$ & 1345 & 5861 & 5336 & $51(2)$ \\
$\mathrm{H}(24 \mathrm{C})$ & 613 & 7173 & 5484 & $51(2)$ \\
$\mathrm{H}(26 \mathrm{~A})$ & 1389 & 16560 & 7343 & $51(2)$ \\
$\mathrm{H}(26 \mathrm{~B})$ & 1471 & 18111 & 7822 & $51(2)$ \\
$\mathrm{H}(26 \mathrm{C})$ & 653 & 16911 & 7875 & $51(2)$ \\
$\mathrm{H}(27 \mathrm{~A})$ & 1062 & 16658 & 9144 & $51(2)$ \\
$\mathrm{H}(27 \mathrm{~B})$ & 1895 & 17823 & 9090 & $51(2)$ \\
$\mathrm{H}(27 \mathrm{C})$ & 2044 & 16083 & 9413 & $51(2)$ \\
$\mathrm{H}(28 \mathrm{~A})$ & 3171 & 15540 & 8572 & $51(2)$ \\
& & & &
\end{tabular}




$\begin{array}{lllll}\mathrm{H}(28 \mathrm{~B}) & 3012 & 17233 & 8206 & 51(2) \\ \mathrm{H}(28 \mathrm{C}) & 2893 & 15636 & 7760 & 51(2)\end{array}$

\section{Structure Determination.}

Orange plates of flor $\mathbf{2}\left(\mathbf{7}(\mathbf{P h N i t})_{2}\right)$ were crystallized from methanol/pentane at $23 \mathrm{deg}$. C. A crystal of dimensions $0.24 \times 0.22 \times 0.20 \mathrm{~mm}$ was mounted on a standard Bruker SMART CCD-based X-ray diffractometer equipped with a LT-2 low temperature device and normal focus Mo-target X-ray tube $(\lambda=0.71073 \mathrm{~A})$ operated at $2000 \mathrm{~W}$ power $(50 \mathrm{kV}, 40 \mathrm{~mA})$. The X-ray intensities were measured at $158(2) \mathrm{K}$; the detector was placed at a distance $5.008 \mathrm{~cm}$ from the crystal. A total of 3332 frames were collected with a scan width of $0.3^{\circ}$ in $\omega$ and phi with an exposure time of $75 \mathrm{~s} /$ frame. The frames were integrated with the Bruker SAINT software package with a narrow frame algorithm. The integration of the data yielded a total of 45865 reflections to a maximum $2 \theta$ value of $42.0^{\circ}$ of which 3007 were independent and 1979 were greater than $2 \sigma(\mathrm{I})$. The final cell constants (Table 1) were based on the xyz centroids of 5901 reflections above $10 \sigma(\mathrm{I})$. Analysis of the data showed negligible decay during data collection. The data were processed with SADABS, no correction for absorption was necessary. The structure was solved and refined with the Bruker SHELXTL (version 5.10) software package using the space group Pbca with $\mathrm{Z}=8$ for the formula $\mathrm{C}_{33} \mathrm{H}_{34} \mathrm{~N}_{2} \mathrm{O}_{2}$. All non-hydrogen atoms were refined anisotropically with the hydrogen atoms placed in idealized positions. Full matrix least-squares refinement based on $\mathrm{F}^{2}$ converged at R1 = 0.0708 and wR2 $=0.1737$ [based on $\mathrm{I}>2 \operatorname{sigma}(\mathrm{I})], \mathrm{R} 1=0.1010$ and $\mathrm{wR} 2=0.1896$ for all data. Additional details are presented in Table 1 and are given as Supporting Information in a CIF file.

Sheldrick, G.M. SHELXTL, v. 5.10; Bruker Analytical X-ray, Madison, WI, 1997.

Sheldrick, G.M. SADABS. Program for Empirical Absorption Correction of Area Detector Data, University of Gottingen: Gottingen, Germany, 1996.

Saint Plus, v. 6.02, Bruker Analytical X-ray, Madison, WI, 1999.

Table S16. Crystal data and structure refinement for flor $2\left(\mathbf{7}(\mathbf{P h N i t})_{2}\right)$.

Identification code

Empirical formula

Formula weight

Temperature

Wavelength

Crystal system, space group

Unit cell dimensions

Volume

Z, Calculated density

Absorption coefficient

$\mathrm{F}(000)$

Crystal size

Theta range for data collection

Limiting indices

Reflections collected / unique

Completeness to theta $=21.00$

Absorption correction

Refinement method

Data / restraints / parameters

Goodness-of-fit on $\mathrm{F}^{\wedge} 2$

Final $\mathrm{R}$ indices [I $>2 \operatorname{sigma}(\mathrm{I})]$

$\mathrm{R}$ indices (all data)

Extinction coefficient

Largest diff. peak and hole

Table S17. Atomic coordinates ( x 10^4) and equivalent isotropic displacement parameters $\left(\mathrm{A}^{\wedge} 2\right.$ x $\left.10^{\wedge} 3\right)$ for flor 2 $\left(\mathbf{7}(\mathbf{P h N i t})_{2}\right)$.

$\mathrm{U}(\mathrm{eq})$ is defined as one third of the trace of the orthogonalized Uij tensor.

\begin{tabular}{llccc}
\hline $\mathrm{x}$ & $\mathrm{y}$ & $\mathrm{z}$ & $\mathrm{U}(\mathrm{eq})$ \\
& \multicolumn{4}{l}{} \\
\hline $\mathrm{N}(1)$ & $5492(3)$ & $1884(2)$ & $7226(2)$ & $46(1)$ \\
$\mathrm{N}(2)$ & $7992(3)$ & $690(2)$ & $3502(2)$ & $50(1)$
\end{tabular}

flor2

34 N2 O 2

$158(2) \mathrm{K}$

$0.71073 \mathrm{~A}$

Orthorhombic, Pbca

$\mathrm{a}=12.087(5) \mathrm{A}$ alpha $=90 \mathrm{deg}$.

$\mathrm{b}=19.740(9) \mathrm{A} \quad$ beta $=90 \mathrm{deg}$.

$\mathrm{c}=23.655(10) \mathrm{A}$ gamma $=90 \mathrm{deg}$.

5644(4) $\mathrm{A}^{\wedge} 3$

$8,1.155 \mathrm{Mg} / \mathrm{m}^{\wedge} 3$

2096

$0.20 \times 0.22 \times 0.24 \mathrm{~mm}$

1.72 to $21.00 \mathrm{deg}$.

$-12<=\mathrm{h}<=12,-19<=\mathrm{k}<=19,-23<=\mathrm{l}<=23$

$45865 / 3007$ [R(int) $=0.0885]$

$99.3 \%$

None

Full-matrix least-squares on $\mathrm{F}^{\wedge} 2$

3007 / 0 / 342

0.966

$\mathrm{R} 1=0.0718, \mathrm{wR} 2=0.1737$

$\mathrm{R} 1=0.1010, \mathrm{wR} 2=0.1896$

$0.0078(11)$

0.305 and -0.304 e. $\mathrm{A}^{\wedge} \mathrm{-}-3$ 


$\begin{array}{lcccc}\mathrm{O}(1) & 6286(3) & 1469(2) & 7382(2) & 79(1) \\ \mathrm{O}(2) & 8813(3) & 1115(2) & 3540(2) & 77(1) \\ \mathrm{C}(1) & 5333(4) & 2512(2) & 7604(2) & 45(1) \\ \mathrm{C}(2) & 4091(4) & 2612(3) & 7730(2) & 66(2) \\ \mathrm{C}(3) & 5848(4) & 3133(2) & 7299(2) & 56(1) \\ \mathrm{C}(4) & 5954(4) & 2386(2) & 8161(2) & 57(1) \\ \mathrm{C}(5) & 5040(3) & 1772(2) & 6677(2) & 38(1) \\ \mathrm{C}(6) & 5347(3) & 1166(2) & 6387(2) & 39(1) \\ \mathrm{C}(7) & 5074(3) & 1082(2) & 5819(2) & 38(1) \\ \mathrm{C}(8) & 4459(3) & 1574(2) & 5513(2) & 38(1) \\ \mathrm{C}(9) & 4078(3) & 2138(2) & 5835(2) & 39(1) \\ \mathrm{C}(10) & 4347(3) & 2235(2) & 6395(2) & 38(1) \\ \mathrm{C}(11) & 4216(3) & 1503(2) & 4878(2) & 37(1) \\ \mathrm{C}(12) & 5207(3) & 1213(2) & 4538(2) & 38(1) \\ \mathrm{C}(13) & 6303(4) & 1432(2) & 4660(2) & 41(1) \\ \mathrm{C}(14) & 7195(3) & 1246(2) & 4321(2) & 43(1) \\ \mathrm{C}(15) & 7037(3) & 839(2) & 3839(2) & 40(1) \\ \mathrm{C}(16) & 5962(3) & 613(2) & 3711(2) & 43(1) \\ \mathrm{C}(17) & 5066(3) & 806(2) & 4052(2) & 40(1) \\ \mathrm{C}(18) & 8171(4) & 56(2) & 3144(2) & 47(1) \\ \mathrm{C}(19) & 7604(4) & 135(2) & 2575(2) & 72(2) \\ \mathrm{C}(20) & 7758(4) & -575(2) & 3478(2) & 62(2) \\ \mathrm{C}(21) & 9432(4) & -21(2) & 3048(2) & 70(2) \\ \mathrm{C}(22) & 3912(4) & 2198(2) & 4597(2) & 40(1) \\ \mathrm{C}(23) & 4561(4) & 2781(2) & 4564(2) & 47(1) \\ \mathrm{C}(24) & 4119(4) & 3355(2) & 4287(2) & 52(1) \\ \mathrm{C}(25) & 3074(4) & 3329(2) & 4042(2) & 51(1) \\ \mathrm{C}(26) & 2422(4) & 2745(2) & 4072(2) & 48(1) \\ \mathrm{C}(27) & 2854(3) & 2172(2) & 4354(2) & 42(1) \\ \mathrm{C}(28) & 2350(3) & 1495(2) & 4478(2) & 40(1) \\ \mathrm{C}(29) & 1308(4) & 1230(2) & 4340(2) & 49(1) \\ \mathrm{C}(30) & 1050(4) & 569(2) & 4513(2) & 53(1) \\ \mathrm{C}(31) & 1828(4) & 175(2) & 4806(2) & 49(1) \\ \mathrm{C}(32) & 2871(3) & 440(2) & 4944(2) & 44(1) \\ \mathrm{C}(33) & 3138(3) & 1101(2) & 4775(2) & 36(1)\end{array}$

Table S18. Bond lengths [A] and angles [deg] for flor2 (7(PhNit) $\left.)_{2}\right)$.

\begin{tabular}{lc}
\hline $\mathrm{N}(1)-\mathrm{O}(1)$ & $1.316(4)$ \\
$\mathrm{N}(1)-\mathrm{C}(5)$ & $1.426(5)$ \\
$\mathrm{N}(1)-\mathrm{C}(1)$ & $1.540(5)$ \\
$\mathrm{N}(2)-\mathrm{O}(2)$ & $1.303(4)$ \\
$\mathrm{N}(2)-\mathrm{C}(15)$ & $1.434(5)$ \\
$\mathrm{N}(2)-\mathrm{C}(18)$ & $1.526(5)$ \\
$\mathrm{C}(1)-\mathrm{C}(4)$ & $1.537(6)$ \\
$\mathrm{C}(1)-\mathrm{C}(2)$ & $1.543(6)$ \\
$\mathrm{C}(1)-\mathrm{C}(3)$ & $1.553(6)$ \\
$\mathrm{C}(5)-\mathrm{C}(10)$ & $1.408(5)$ \\
$\mathrm{C}(5)-\mathrm{C}(6)$ & $1.428(5)$ \\
$\mathrm{C}(6)-\mathrm{C}(7)$ & $1.393(6)$ \\
$\mathrm{C}(7)-\mathrm{C}(8)$ & $1.420(5)$ \\
$\mathrm{C}(8)-\mathrm{C}(9)$ & $1.426(6)$ \\
$\mathrm{C}(8)-\mathrm{C}(11)$ & $1.539(6)$ \\
$\mathrm{C}(9)-\mathrm{C}(10)$ & $1.378(6)$ \\
$\mathrm{C}(11)-\mathrm{C}(33)$ & $1.544(6)$ \\
$\mathrm{C}(11)-\mathrm{C}(12)$ & $1.553(6)$ \\
$\mathrm{C}(11)-\mathrm{C}(22)$ & $1.567(6)$ \\
$\mathrm{C}(12)-\mathrm{C}(17)$ & $1.412(5)$
\end{tabular}




\begin{tabular}{|c|c|}
\hline C(12)-C(13) & $1.423(6)$ \\
\hline $\mathrm{C}(13)-\mathrm{C}(14)$ & $1.394(6)$ \\
\hline$C(14)-C(15)$ & $1.407(6)$ \\
\hline$C(15)-C(16)$ & $1.407(6)$ \\
\hline$C(16)-C(17)$ & $1.404(6)$ \\
\hline C(18)-C(19) & $1.519(6)$ \\
\hline $\mathrm{C}(18)-\mathrm{C}(21)$ & $1.548(6)$ \\
\hline $\mathrm{C}(18)-\mathrm{C}(20)$ & $1.556(6)$ \\
\hline$C(22)-C(23)$ & $1.396(6)$ \\
\hline $\mathrm{C}(22)-\mathrm{C}(27)$ & $1.402(6)$ \\
\hline $\mathrm{C}(23)-\mathrm{C}(24)$ & $1.413(6)$ \\
\hline $\mathrm{C}(24)-\mathrm{C}(25)$ & $1.391(6)$ \\
\hline$C(25)-C(26)$ & $1.399(6)$ \\
\hline$C(26)-C(27)$ & $1.414(6)$ \\
\hline $\mathrm{C}(27)-\mathrm{C}(28)$ & $1.496(6)$ \\
\hline $\mathrm{C}(28)-\mathrm{C}(29)$ & $1.403(6)$ \\
\hline $\mathrm{C}(28)-\mathrm{C}(33)$ & $1.418(6)$ \\
\hline C(29)-C(30) & $1.402(6)$ \\
\hline $\mathrm{C}(30)-\mathrm{C}(31)$ & $1.404(6)$ \\
\hline $\mathrm{C}(31)-\mathrm{C}(32)$ & $1.404(6)$ \\
\hline $\mathrm{C}(32)-\mathrm{C}(33)$ & $1.403(5)$ \\
\hline $\mathrm{O}(1)-\mathrm{N}(1)-\mathrm{C}(5)$ & $116.0(3)$ \\
\hline $\mathrm{O}(1)-\mathrm{N}(1)-\mathrm{C}(1)$ & $115.4(3)$ \\
\hline $\mathrm{C}(5)-\mathrm{N}(1)-\mathrm{C}(1)$ & $127.3(3)$ \\
\hline $\mathrm{O}(2)-\mathrm{N}(2)-\mathrm{C}(15)$ & $116.3(3)$ \\
\hline $\mathrm{O}(2)-\mathrm{N}(2)-\mathrm{C}(18)$ & $117.3(3)$ \\
\hline $\mathrm{C}(15)-\mathrm{N}(2)-\mathrm{C}(18)$ & $126.3(3)$ \\
\hline $\mathrm{C}(4)-\mathrm{C}(1)-\mathrm{N}(1)$ & $107.8(3)$ \\
\hline $\mathrm{C}(4)-\mathrm{C}(1)-\mathrm{C}(2)$ & $109.2(4)$ \\
\hline $\mathrm{N}(1)-\mathrm{C}(1)-\mathrm{C}(2)$ & $109.7(4)$ \\
\hline $\mathrm{C}(4)-\mathrm{C}(1)-\mathrm{C}(3)$ & $109.3(4)$ \\
\hline $\mathrm{N}(1)-\mathrm{C}(1)-\mathrm{C}(3)$ & $108.4(3)$ \\
\hline $\mathrm{C}(2)-\mathrm{C}(1)-\mathrm{C}(3)$ & $112.3(4)$ \\
\hline $\mathrm{C}(10)-\mathrm{C}(5)-\mathrm{N}(1)$ & $123.9(4)$ \\
\hline$C(10)-C(5)-C(6)$ & $118.0(4)$ \\
\hline $\mathrm{N}(1)-\mathrm{C}(5)-\mathrm{C}(6)$ & $118.0(4)$ \\
\hline$C(7)-C(6)-C(5)$ & $120.2(4)$ \\
\hline $\mathrm{C}(6)-\mathrm{C}(7)-\mathrm{C}(8)$ & $122.2(4)$ \\
\hline $\mathrm{C}(7)-\mathrm{C}(8)-\mathrm{C}(9)$ & $115.6(4)$ \\
\hline$C(7)-C(8)-C(11)$ & $122.4(4)$ \\
\hline $\mathrm{C}(9)-\mathrm{C}(8)-\mathrm{C}(11)$ & $122.1(4)$ \\
\hline $\mathrm{C}(10)-\mathrm{C}(9)-\mathrm{C}(8)$ & $123.1(4)$ \\
\hline $\mathrm{C}(9)-\mathrm{C}(10)-\mathrm{C}(5)$ & $120.4(4)$ \\
\hline $\mathrm{C}(8)-\mathrm{C}(11)-\mathrm{C}(33)$ & $111.2(3)$ \\
\hline $\mathrm{C}(8)-\mathrm{C}(11)-\mathrm{C}(12)$ & 113.1(3) \\
\hline$C(33)-C(11)-C(12)$ & $112.3(3)$ \\
\hline $\mathrm{C}(8)-\mathrm{C}(11)-\mathrm{C}(22)$ & $112.3(3)$ \\
\hline$C(33)-C(11)-C(22)$ & $100.7(3)$ \\
\hline $\mathrm{C}(12)-\mathrm{C}(11)-\mathrm{C}(22)$ & $106.5(3)$ \\
\hline $\mathrm{C}(17)-\mathrm{C}(12)-\mathrm{C}(13)$ & $116.8(4)$ \\
\hline $\mathrm{C}(17)-\mathrm{C}(12)-\mathrm{C}(11)$ & $122.5(4)$ \\
\hline$C(13)-C(12)-C(11)$ & $120.0(4)$ \\
\hline$C(14)-C(13)-C(12)$ & $121.5(4)$ \\
\hline $\mathrm{C}(13)-\mathrm{C}(14)-\mathrm{C}(15)$ & $120.8(4)$ \\
\hline $\mathrm{C}(16)-\mathrm{C}(15)-\mathrm{C}(14)$ & $118.8(4)$ \\
\hline $\mathrm{C}(16)-\mathrm{C}(15)-\mathrm{N}(2)$ & $123.9(4)$ \\
\hline $\mathrm{C}(14)-\mathrm{C}(15)-\mathrm{N}(2)$ & $117.3(4)$ \\
\hline$C(17)-C(16)-C(15)$ & $120.1(4)$ \\
\hline
\end{tabular}




$\begin{array}{lc}\mathrm{C}(16)-\mathrm{C}(17)-\mathrm{C}(12) & 121.9(4) \\ \mathrm{C}(19)-\mathrm{C}(18)-\mathrm{N}(2) & 110.1(3) \\ \mathrm{C}(19)-\mathrm{C}(18)-\mathrm{C}(21) & 108.9(4) \\ \mathrm{N}(2)-\mathrm{C}(18)-\mathrm{C}(21) & 107.6(3) \\ \mathrm{C}(19)-\mathrm{C}(18)-\mathrm{C}(20) & 112.7(4) \\ \mathrm{N}(2)-\mathrm{C}(18)-\mathrm{C}(20) & 109.2(4) \\ \mathrm{C}(21)-\mathrm{C}(18)-\mathrm{C}(20) & 108.2(4) \\ \mathrm{C}(23)-\mathrm{C}(22)-\mathrm{C}(27) & 121.4(4) \\ \mathrm{C}(23)-\mathrm{C}(22)-\mathrm{C}(11) & 127.8(4) \\ \mathrm{C}(27)-\mathrm{C}(22)-\mathrm{C}(11) & 110.8(4) \\ \mathrm{C}(22)-\mathrm{C}(23)-\mathrm{C}(24) & 118.3(4) \\ \mathrm{C}(25)-\mathrm{C}(24)-\mathrm{C}(23) & 120.5(4) \\ \mathrm{C}(24)-\mathrm{C}(25)-\mathrm{C}(26) & 121.3(4) \\ \mathrm{C}(25)-\mathrm{C}(26)-\mathrm{C}(27) & 118.4(4) \\ \mathrm{C}(22)-\mathrm{C}(27)-\mathrm{C}(26) & 120.1(4) \\ \mathrm{C}(22)-\mathrm{C}(27)-\mathrm{C}(28) & 109.0(4) \\ \mathrm{C}(26)-\mathrm{C}(27)-\mathrm{C}(28) & 130.9(4) \\ \mathrm{C}(29)-\mathrm{C}(28)-\mathrm{C}(33) & 120.9(4) \\ \mathrm{C}(29)-\mathrm{C}(28)-\mathrm{C}(27) & 130.9(4) \\ \mathrm{C}(33)-\mathrm{C}(28)-\mathrm{C}(27) & 108.2(3) \\ \mathrm{C}(30)-\mathrm{C}(29)-\mathrm{C}(28) & 118.6(4) \\ \mathrm{C}(29)-\mathrm{C}(30)-\mathrm{C}(31) & 120.8(4) \\ \mathrm{C}(30)-\mathrm{C}(31)-\mathrm{C}(32) & 120.6(4) \\ \mathrm{C}(33)-\mathrm{C}(32)-\mathrm{C}(31) & 119.2(4) \\ \mathrm{C}(32)-\mathrm{C}(33)-\mathrm{C}(28) & 119.8(4) \\ \mathrm{C}(32)-\mathrm{C}(33)-\mathrm{C}(11) & 128.9(4) \\ \mathrm{C}(28)-\mathrm{C}(33)-\mathrm{C}(11) & 111.2(3) \\ \end{array}$

Symmetry transformations used to generate equivalent atoms:

Table S19. Anisotropic displacement parameters $\left(\mathrm{A}^{\wedge} 2 \times 10^{\wedge} 3\right)$ for flor $2\left(\mathbf{7}(\mathbf{P h N i t})_{2}\right)$.

The anisotropic displacement factor exponent takes the form: $-2 \mathrm{pi}^{\wedge} 2$ [ $\mathrm{h}^{\wedge} 2 \mathrm{a}^{* \wedge} 2 \mathrm{U} 11+\ldots+2 \mathrm{~h} \mathrm{k} \mathrm{a} \mathrm{b}^{*} \mathrm{U} 12$ ]

\begin{tabular}{lcccccc}
\hline $\mathrm{U} 11$ & $\mathrm{U} 22$ & $\mathrm{U} 33$ & $\mathrm{U} 23$ & $\mathrm{U} 13$ & $\mathrm{U} 12$ \\
& & & & & & \\
$\mathrm{~N}(1)$ & $43(2)$ & $25(2)$ & $70(3)$ & $2(2)$ & $-4(2)$ & $7(2)$ \\
$\mathrm{N}(2)$ & $32(2)$ & $28(2)$ & $89(3)$ & $-11(2)$ & $14(2)$ & $-6(2)$ \\
$\mathrm{O}(1)$ & $90(3)$ & $49(2)$ & $97(3)$ & $-10(2)$ & $-28(2)$ & $30(2)$ \\
$\mathrm{O}(2)$ & $46(2)$ & $45(2)$ & $138(3)$ & $-21(2)$ & $31(2)$ & $-24(2)$ \\
$\mathrm{C}(1)$ & $37(3)$ & $36(3)$ & $63(3)$ & $-6(2)$ & $6(2)$ & $2(2)$ \\
$\mathrm{C}(2)$ & $50(4)$ & $72(4)$ & $75(4)$ & $-4(3)$ & $6(3)$ & $9(3)$ \\
$\mathrm{C}(3)$ & $37(3)$ & $35(3)$ & $94(4)$ & $1(2)$ & $-6(3)$ & $-6(2)$ \\
$\mathrm{C}(4)$ & $57(3)$ & $48(3)$ & $66(3)$ & $-9(2)$ & $-6(3)$ & $3(3)$ \\
$\mathrm{C}(5)$ & $27(3)$ & $29(3)$ & $59(3)$ & $5(2)$ & $-2(2)$ & $2(2)$ \\
$\mathrm{C}(6)$ & $30(3)$ & $20(2)$ & $66(3)$ & $4(2)$ & $2(2)$ & $2(2)$ \\
$\mathrm{C}(7)$ & $26(3)$ & $21(2)$ & $69(3)$ & $0(2)$ & $1(2)$ & $-1(2)$ \\
$\mathrm{C}(8)$ & $19(2)$ & $23(2)$ & $71(3)$ & $2(2)$ & $3(2)$ & $-1(2)$ \\
$\mathrm{C}(9)$ & $27(3)$ & $27(2)$ & $64(3)$ & $3(2)$ & $5(2)$ & $9(2)$ \\
$\mathrm{C}(10)$ & $23(2)$ & $29(2)$ & $62(3)$ & $-5(2)$ & $6(2)$ & $1(2)$ \\
$\mathrm{C}(11)$ & $26(3)$ & $23(2)$ & $62(3)$ & $2(2)$ & $1(2)$ & $6(2)$ \\
$\mathrm{C}(12)$ & $30(3)$ & $22(2)$ & $62(3)$ & $1(2)$ & $-1(2)$ & $5(2)$ \\
$\mathrm{C}(13)$ & $33(3)$ & $22(2)$ & $68(3)$ & $-3(2)$ & $-3(2)$ & $1(2)$ \\
$\mathrm{C}(14)$ & $26(3)$ & $25(2)$ & $79(3)$ & $2(2)$ & $-1(2)$ & $0(2)$ \\
$\mathrm{C}(15)$ & $29(3)$ & $24(2)$ & $68(3)$ & $-1(2)$ & $5(2)$ & $1(2)$ \\
$\mathrm{C}(16)$ & $33(3)$ & $24(2)$ & $71(3)$ & $-7(2)$ & $0(2)$ & $1(2)$ \\
$\mathrm{C}(17)$ & $27(3)$ & $29(2)$ & $65(3)$ & $-1(2)$ & $-1(2)$ & $1(2)$ \\
$\mathrm{C}(18)$ & $35(3)$ & $33(3)$ & $74(3)$ & $-6(2)$ & $10(2)$ & $2(2)$ \\
$\mathrm{C}(19)$ & $73(4)$ & $63(3)$ & $81(4)$ & $-14(3)$ & $7(3)$ & $13(3)$
\end{tabular}




$\begin{array}{llllrrr}\mathrm{C}(20) & 53(3) & 32(3) & 101(4) & -10(2) & 20(3) & -1(2) \\ \mathrm{C}(21) & 43(3) & 53(3) & 115(4) & -17(3) & 15(3) & 4(3) \\ \mathrm{C}(22) & 33(3) & 28(3) & 60(3) & -5(2) & 2(2) & 7(2) \\ \mathrm{C}(23) & 33(3) & 30(3) & 77(3) & -3(2) & 5(2) & 3(2) \\ \mathrm{C}(24) & 44(3) & 27(3) & 84(3) & 1(2) & 8(3) & 10(2) \\ \mathrm{C}(25) & 44(3) & 34(3) & 74(3) & 4(2) & 6(3) & 13(3) \\ \mathrm{C}(26) & 34(3) & 35(3) & 74(3) & 2(2) & -2(2) & 18(2) \\ \mathrm{C}(27) & 28(3) & 36(3) & 63(3) & -6(2) & 1(2) & 8(2) \\ \mathrm{C}(28) & 24(3) & 30(3) & 65(3) & -3(2) & 1(2) & 2(2) \\ \mathrm{C}(29) & 32(3) & 45(3) & 71(3) & -4(2) & -4(2) & 6(2) \\ \mathrm{C}(30) & 28(3) & 45(3) & 85(4) & -4(3) & -4(3) & -7(2) \\ \mathrm{C}(31) & 35(3) & 31(3) & 79(3) & 1(2) & 3(3) & -11(3) \\ \mathrm{C}(32) & 31(3) & 31(3) & 72(3) & -4(2) & 2(2) & -5(2) \\ \mathrm{C}(33) & 23(3) & 23(3) & 63(3) & -4(2) & 4(2) & 0(2)\end{array}$

Table S20. Hydrogen coordinates ( $\left.\mathrm{x} 10^{\wedge} 4\right)$ and isotropic displacement parameters $\left(\mathrm{A}^{\wedge} 2 \times 10^{\wedge} 3\right)$ for flor2 $\left(\mathbf{7}(\mathbf{P h N i t})_{2}\right)$.

\begin{tabular}{lcccc}
\hline & $\mathrm{y}$ & \multicolumn{3}{c}{$\mathrm{U}(\mathrm{eq})$} \\
\hline $\mathrm{H}(2 \mathrm{~A})$ & 3728 & 2813 & 7400 & $70(3)$ \\
$\mathrm{H}(2 \mathrm{~B})$ & 4005 & 2914 & 8056 & $70(3)$ \\
$\mathrm{H}(2 \mathrm{C})$ & 3752 & 2172 & 7815 & $70(3)$ \\
$\mathrm{H}(3 \mathrm{~A})$ & 6624 & 3038 & 7209 & $70(3)$ \\
$\mathrm{H}(3 \mathrm{~B})$ & 5805 & 3531 & 7546 & $70(3)$ \\
$\mathrm{H}(3 \mathrm{C})$ & 5440 & 3223 & 6949 & $70(3)$ \\
$\mathrm{H}(4 \mathrm{~A})$ & 5659 & 1978 & 8343 & $70(3)$ \\
$\mathrm{H}(4 \mathrm{~B})$ & 5855 & 2776 & 8412 & $70(3)$ \\
$\mathrm{H}(4 \mathrm{C})$ & 6743 & 2323 & 8082 & $70(3)$ \\
$\mathrm{H}(6)$ & 5738 & 820 & 6581 & $70(3)$ \\
$\mathrm{H}(7)$ & 5307 & 682 & 5629 & $70(3)$ \\
$\mathrm{H}(9)$ & 3616 & 2462 & 5655 & $70(3)$ \\
$\mathrm{H}(10)$ & 4064 & 2617 & 6592 & $70(3)$ \\
$\mathrm{H}(13)$ & 6429 & 1712 & 4981 & $70(3)$ \\
$\mathrm{H}(14)$ & 7918 & 1395 & 4416 & $70(3)$ \\
$\mathrm{H}(16)$ & 5843 & 329 & 3392 & $70(3)$ \\
$\mathrm{H}(17)$ & 4343 & 658 & 3954 & $70(3)$ \\
$\mathrm{H}(19 \mathrm{~A})$ & 6803 & 169 & 2631 & $70(3)$ \\
$\mathrm{H}(19 \mathrm{~B})$ & 7770 & -259 & 2338 & $70(3)$ \\
$\mathrm{H}(19 \mathrm{C})$ & 7874 & 546 & 2389 & $70(3)$ \\
$\mathrm{H}(20 \mathrm{~A})$ & 8114 & -587 & 3850 & $70(3)$ \\
$\mathrm{H}(20 \mathrm{~B})$ & 7946 & -987 & 3268 & $70(3)$ \\
$\mathrm{H}(20 \mathrm{C})$ & 6954 & -548 & 3525 & $70(3)$ \\
$\mathrm{H}(21 \mathrm{~A})$ & 9712 & 376 & 2845 & $70(3)$ \\
$\mathrm{H}(21 \mathrm{~B})$ & 9574 & -430 & 2824 & $70(3)$ \\
$\mathrm{H}(21 \mathrm{C})$ & 9807 & -60 & 3414 & $70(3)$ \\
$\mathrm{H}(23)$ & 5281 & 2793 & 4725 & $70(3)$ \\
$\mathrm{H}(24)$ & 4539 & 3761 & 4268 & $70(3)$ \\
$\mathrm{H}(25)$ & 2798 & 3716 & 3850 & $70(3)$ \\
$\mathrm{H}(26)$ & 1705 & 2733 & 3908 & $70(3)$ \\
$\mathrm{H}(29)$ & 787 & 1492 & 4134 & $70(3)$ \\
$\mathrm{H}(30)$ & 341 & 386 & 4431 & $70(3)$ \\
$\mathrm{H}(31)$ & 1646 & -276 & 4912 & $70(3)$ \\
$\mathrm{H}(32)$ & 3389 & 175 & 5150 & $70(3)$ \\
\hline $\mathrm{Str}$ & & & & \\
\hline
\end{tabular}

\section{Structure Determination.}

Crystal data of a red plate-like crystal of $\mathbf{3}(\mathbf{P h N i t})_{2}$ with the approximate dimensions $0.20 \times 0.20 \times 0.05 \mathrm{~mm}$ were collected on a Bruker platform diffractometer equipped with a Smart6000 CCD detectori (normal focus sealed 
tube operated at $50 \mathrm{kV}, 45 \mathrm{~mA}$, graphite-monochromated, MoK $\alpha=0.71073 \AA$ ). A single crystal was selected and mounted on a $0.1 \mathrm{~mm}$ glass capillary with epoxy glue. The crystal was measured at ca. 25(5) K using an open flow He-cryostat ${ }^{\text {ii. }}$ Evaluation of the diffraction data revealed cracking for all tested crystals into several components upon cooling. A total of 1073 frames were collected with an exposure time of $60 \mathrm{~s}$ and a scan width of $0.3^{\circ}$ in _ at a detector to crystal distance of $5.150 \mathrm{~cm}$.

Orientation Matrices for four components were found using Gemini iii. The data for all four crystal components were integrated simultaneously using SAINT $6.29 \mathrm{~A}^{\mathrm{iv}}$. No correction for absorption or decay was applied. A separate integration based on only the orientation matrix of the major component (and thereby containing all reflections originated by component 1 - isolated and overlapping) provided a 'HKLF 4' format file, which was used to solve the structure with direct methods in P1bar (SHELXTL 5.1 ${ }^{v}$ ). The structure was refined by full-matrix least squares on $\mathrm{F}^{2}$. A refinement on all four components similtaneously converged to $\mathrm{R} 1=0.184\left(48^{\circ}\right.$ cutoff in 2_) for 10364 reflections with Fo > 4_(Fo) and resulted in batch scale factors of 0.57: 0.20:0.21:0.02. All non-hydrogen atoms were refined with isotropic atomic displacement factors, hydrogen atoms were calculated on idealized positions and treated as riding atoms.

The high R-value is not surprising considering the nature of this cracked crystal, which can be only approximated by a model of four single crystal domains. Not all 'domains' in the cracked crystal are accounted for and some of the indexed ones are obviously not well defined. A home written program was used to separate overlapping and isolated reflections and a better model was obtained by refining on those reflections that originated only from the dominant component. Nitrogen and Oxygen atoms were refined with anisotropic atomic displacement factors, carbon atom were refined isotropically, hydrogen atoms were calculated on idealized positions and treated as riding atoms. The final refinement converged to $\mathrm{R} 1=0.0948(\mathrm{wR} 2=0.2259)$ for 1129 reflections with Fo > 4_(Fo) and $\mathrm{R} 1=0.1031(\mathrm{wR} 2=0.2323)$ for all 1254 data.

Table S21. Crystal data and structure refinement for abcd3a (3(PhNit $\left.)_{2}\right)$.

\author{
Empirical formula \\ Formula weight \\ Temperature \\ Wavelength \\ Crystal system, space group \\ Unit cell dimensions
}

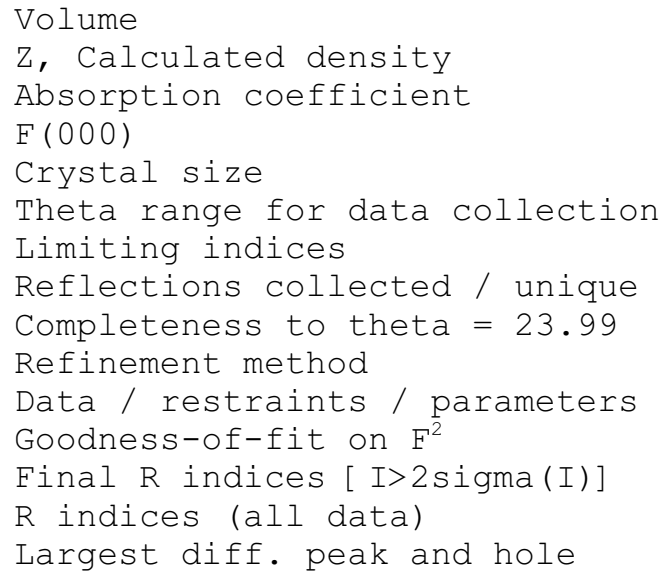

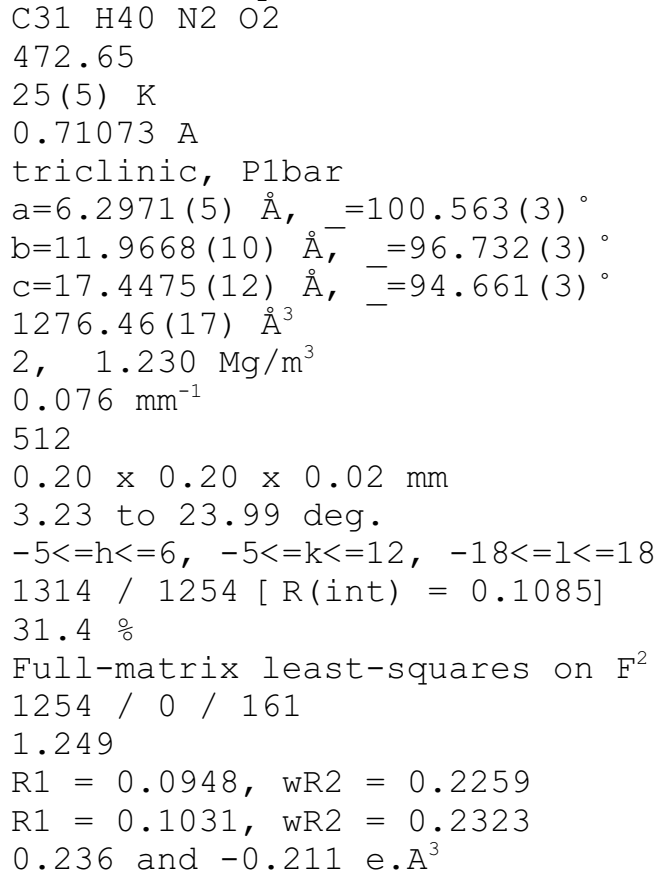

Table S22. Atomic coordinates $\left(\times 10^{4}\right)$ and equivalent isotropic or isotropic displacement parameters $\left(\AA^{2} \times 10^{3}\right)$ for abcd3a $\left(\mathbf{3}(\mathbf{P h N i t})_{2}\right)$. U(eq) is defined as one third of the trace of the orthogonalized Uij tensor.

\begin{tabular}{lcccc}
\hline \multicolumn{1}{c}{$\mathrm{x}$} & $\mathrm{y}$ & $\mathrm{Z}$ & $\mathrm{U}(\mathrm{eq})$ & \\
\hline $\mathrm{O}(1)$ & $-25036(10)$ & $-11168(6)$ & $-7226(3)$ & $14(2)$ \\
$\mathrm{O}(2)$ & $-13700(10)$ & $-15740(6)$ & $-5370(3)$ & $12(2)$ \\
$\mathrm{N}(1)$ & $-23054(12)$ & $-11248(7)$ & $-7331(3)$ & $12(2)$ \\
$\mathrm{N}(2)$ & $-15630(13)$ & $-16155(7)$ & $-5305(3)$ & $16(2)$
\end{tabular}




\begin{tabular}{|c|c|c|c|c|}
\hline$C(1)$ & $-21606(15)$ & $-10128(9)$ & $-7148(4)$ & $14(2)$ \\
\hline$C(2)$ & $-20018(16)$ & $-10100(9)$ & $-7753(4)$ & $20(2)$ \\
\hline$C(3)$ & $-23023(15)$ & $-9167(9)$ & $-7177(4)$ & $15(2)$ \\
\hline C (4) & $-20379(15)$ & $-9994(9)$ & $-6304(4)$ & $15(2)$ \\
\hline C ( 5$)$ & $-22405(14)$ & $-12331(8)$ & $-7571(4)$ & $12(2)$ \\
\hline$C(6)$ & $-20309(14)$ & $-12622(8)$ & $-7441(4)$ & $12(2)$ \\
\hline$C(7)$ & $-19874(15)$ & $-13734(8)$ & $-7637(4)$ & $12(2)$ \\
\hline$C(8)$ & $-21514(14)$ & $-14622(8)$ & $-7993(3)$ & $8(2)$ \\
\hline C (9) & $-23603(14)$ & $-14322(8)$ & $-8128(4)$ & $9(2)$ \\
\hline$C(10)$ & $-24070(15)$ & $-13211(8)$ & $-7929(4)$ & $12(2)$ \\
\hline C (11) & $-21024(14)$ & $-15836(8)$ & $-8123(4)$ & $12(2)$ \\
\hline $\mathrm{C}(12)$ & $-19755(14)$ & $-16146(8)$ & $-7419(3)$ & $7(2)$ \\
\hline C (13) & $-20510(14)$ & $-15862(8)$ & $-6682(4)$ & $12(2)$ \\
\hline$C(14)$ & $-19209(14)$ & $-15959(8)$ & $-5994(4)$ & $10(2)$ \\
\hline$C(15)$ & $-17181(15)$ & $-16284(8)$ & $-6022(4)$ & $11(2)$ \\
\hline$C(16)$ & $-16460(15)$ & $-16619(8)$ & $-6751(4)$ & $14(2)$ \\
\hline C (17) & $-17791(14)$ & $-16558(8)$ & $-7441(4)$ & $9(2)$ \\
\hline $\mathrm{C}(18)$ & $-15833(14)$ & $-16976(8)$ & $-4750(4)$ & $10(2)$ \\
\hline$C(19)$ & $-18142(14)$ & $-17169(8)$ & $-4584(4)$ & $13(2)$ \\
\hline$C(20)$ & $-14342(14)$ & $-16479(8)$ & $-3991(4)$ & $13(2)$ \\
\hline$C(21)$ & $-15090(14)$ & $-18105(8)$ & $-5150(4)$ & $14(2)$ \\
\hline C (22) & $-21609(14)$ & $-16589(8)$ & $-8825(4)$ & $10(2)$ \\
\hline C (23) & $-21461(14)$ & $-17860(8)$ & $-8930(4)$ & $11(2)$ \\
\hline$C(24)$ & $-19878(14)$ & $-18207(8)$ & $-9538(4)$ & $12(2)$ \\
\hline$C(25)$ & $-20705(14)$ & $-17843(8)$ & $-10315(4)$ & $10(2)$ \\
\hline$C(26)$ & $-20862(15)$ & $-16568(8)$ & $-10177(4)$ & $12(2)$ \\
\hline$C(27)$ & $-22461(14)$ & $-16215(8)$ & $-9584(3)$ & $10(2)$ \\
\hline $\mathrm{C}(28)$ & $-24673(14)$ & $-16877(8)$ & $-9907(4)$ & $13(2)$ \\
\hline$C(29)$ & $-24530(15)$ & $-18153(8)$ & $-10039(4)$ & $13(2)$ \\
\hline$C(30)$ & $-22947(14)$ & $-18497(8)$ & $-10635(4)$ & $13(2)$ \\
\hline C (31) & $-23692(14)$ & $-18487(8)$ & $-9250(3)$ & $10(2)$ \\
\hline
\end{tabular}

Table S23. Bond lengths $[\AA]$ and angles $\left[^{\circ}\right]$ for abcd3a $\left(\mathbf{3}(\mathbf{P h N i t})_{2}\right)$.

\begin{tabular}{ll}
\hline$O(1)-\mathrm{N}(1)$ & $1.292(9)$ \\
$O(2)-\mathrm{N}(2)$ & $1.300(9)$ \\
$\mathrm{N}(1)-\mathrm{C}(5)$ & $1.396(12)$ \\
$\mathrm{N}(1)-\mathrm{C}(1)$ & $1.518(12)$ \\
$\mathrm{N}(2)-\mathrm{C}(15)$ & $1.470(10)$ \\
$\mathrm{N}(2)-\mathrm{C}(18)$ & $1.509(13)$ \\
$\mathrm{C}(1)-\mathrm{C}(3)$ & $1.517(15)$ \\
$\mathrm{C}(1)-\mathrm{C}(2)$ & $1.539(12)$ \\
$\mathrm{C}(1)-\mathrm{C}(4)$ & $1.554(10)$ \\
$\mathrm{C}(2)-\mathrm{H}(2 \mathrm{~A})$ & 0.9600 \\
$\mathrm{C}(2)-\mathrm{H}(2 \mathrm{~B})$ & 0.9600 \\
$\mathrm{C}(2)-\mathrm{H}(2 \mathrm{C})$ & 0.9600 \\
$\mathrm{C}(3)-\mathrm{H}(3 \mathrm{~A})$ & 0.9600 \\
$\mathrm{C}(3)-\mathrm{H}(3 \mathrm{~B})$ & 0.9600 \\
$\mathrm{C}(3)-\mathrm{H}(3 \mathrm{C})$ & 0.9600 \\
$\mathrm{C}(4)-\mathrm{H}(4 \mathrm{~A})$ & 0.9600 \\
$\mathrm{C}(4)-\mathrm{H}(4 \mathrm{~B})$ & 0.9600 \\
$\mathrm{C}(4)-\mathrm{H}(4 \mathrm{C})$ & 0.9600 \\
$\mathrm{C}(5)-\mathrm{C}(6)$ & $1.396(14)$ \\
$\mathrm{C}(5)-\mathrm{C}(10)$ & $1.423(12)$ \\
$\mathrm{C}(6)-\mathrm{C}(7)$ & $1.371(13)$ \\
$\mathrm{C}(6)-\mathrm{H}(6 \mathrm{~A})$ & 0.9300
\end{tabular}




\begin{tabular}{|c|c|}
\hline$C(7)-C(8)$ & $1.418(12)$ \\
\hline $\mathrm{C}(7)-\mathrm{H}(7 \mathrm{~A})$ & 0.9300 \\
\hline$C(8)-C(9)$ & $1.396(13)$ \\
\hline$C(8)-C(11)$ & $1.492(13)$ \\
\hline$C(9)-C(10)$ & $1.376(13)$ \\
\hline $\mathrm{C}(9)-\mathrm{H}(9 \mathrm{~A})$ & 0.9300 \\
\hline $\mathrm{C}(10)-\mathrm{H}(10 \mathrm{~A})$ & 0.9300 \\
\hline$C(11)-C(22)$ & $1.372(9)$ \\
\hline$C(11)-C(12)$ & $1.507(12)$ \\
\hline$C(12)-C(17)$ & $1.370(13)$ \\
\hline$C(12)-C(13)$ & $1.415(10)$ \\
\hline$C(13)-C(14)$ & $1.401(12)$ \\
\hline $\mathrm{C}(13)-\mathrm{H}(13 \mathrm{~A})$ & 0.9300 \\
\hline$C(14)-C(15)$ & $1.369(14)$ \\
\hline $\mathrm{C}(14)-\mathrm{H}(14 \mathrm{~A})$ & 0.9300 \\
\hline$C(15)-C(16)$ & $1.401(10)$ \\
\hline$C(16)-C(17)$ & $1.400(12)$ \\
\hline $\mathrm{C}(16)-\mathrm{H}(16 \mathrm{~A})$ & 0.9300 \\
\hline $\mathrm{C}(17)-\mathrm{H}(17 \mathrm{~A})$ & 0.9300 \\
\hline$C(18)-C(20)$ & $1.522(9)$ \\
\hline$C(18)-C(19)$ & $1.523(11)$ \\
\hline$C(18)-C(21)$ & $1.538(13)$ \\
\hline $\mathrm{C}(19)-\mathrm{H}(19 \mathrm{~A})$ & 0.9600 \\
\hline $\mathrm{C}(19)-\mathrm{H}(19 \mathrm{~B})$ & 0.9600 \\
\hline $\mathrm{C}(19)-\mathrm{H}(19 \mathrm{C})$ & 0.9600 \\
\hline $\mathrm{C}(20)-\mathrm{H}(20 \mathrm{~A})$ & 0.9600 \\
\hline$C(20)-H(20 B)$ & 0.9600 \\
\hline $\mathrm{C}(20)-\mathrm{H}(20 \mathrm{C})$ & 0.9600 \\
\hline $\mathrm{C}(21)-\mathrm{H}(21 \mathrm{~A})$ & 0.9600 \\
\hline $\mathrm{C}(21)-\mathrm{H}(21 \mathrm{~B})$ & 0.9600 \\
\hline $\mathrm{C}(21)-\mathrm{H}(21 \mathrm{C})$ & 0.9600 \\
\hline$C(22)-C(23)$ & $1.510(13)$ \\
\hline$C(22)-C(27)$ & $1.528(12)$ \\
\hline$C(23)-C(31)$ & $1.530(12)$ \\
\hline$C(23)-C(24)$ & $1.563(11)$ \\
\hline$C(23)-H(23 A)$ & 0.9800 \\
\hline$C(24)-C(25)$ & $1.544(12)$ \\
\hline $\mathrm{C}(24)-\mathrm{H}(24 \mathrm{~A})$ & 0.9700 \\
\hline $\mathrm{C}(24)-\mathrm{H}(24 \mathrm{~B})$ & 0.9700 \\
\hline$C(25)-C(26)$ & $1.513(13)$ \\
\hline$C(25)-C(30)$ & $1.547(12)$ \\
\hline $\mathrm{C}(25)-\mathrm{H}(25 \mathrm{~A})$ & 0.9800 \\
\hline$C(26)-C(27)$ & $1.554(11)$ \\
\hline $\mathrm{C}(26)-\mathrm{H}(26 \mathrm{~A})$ & 0.9700 \\
\hline$C(26)-H(26 B)$ & 0.9700 \\
\hline$C(27)-C(28)$ & $1.535(12)$ \\
\hline $\mathrm{C}(27)-\mathrm{H}(27 \mathrm{~A})$ & 0.9800 \\
\hline$C(28)-C(29)$ & $1.513(14)$ \\
\hline $\mathrm{C}(28)-\mathrm{H}(28 \mathrm{~A})$ & 0.9700 \\
\hline$C(28)-H(28 B)$ & 0.9700 \\
\hline$C(29)-C(30)$ & $1.548(12)$ \\
\hline$C(29)-C(31)$ & $1.549(12)$ \\
\hline $\mathrm{C}(29)-\mathrm{H}(29 A)$ & 0.9800 \\
\hline $\mathrm{C}(30)-\mathrm{H}(30 \mathrm{~A})$ & 0.9700 \\
\hline$C(30)-H(30 B)$ & 0.9700 \\
\hline $\mathrm{C}(31)-\mathrm{H}(31 \mathrm{~A})$ & 0.9700 \\
\hline $\mathrm{C}(31)-\mathrm{H}(31 \mathrm{~B})$ & 0.9700 \\
\hline $\mathrm{O}(1)-\mathrm{N}(1)-\mathrm{C}(5)$ & $118.7(7)$ \\
\hline
\end{tabular}




\begin{tabular}{|c|c|}
\hline $\mathrm{O}(1)-\mathrm{N}(1)-\mathrm{C}(1)$ & $115.5(7)$ \\
\hline $\mathrm{C}(5)-\mathrm{N}(1)-\mathrm{C}(1)$ & $125.8(7)$ \\
\hline $\mathrm{O}(2)-\mathrm{N}(2)-\mathrm{C}(15)$ & $114.4(6)$ \\
\hline $\mathrm{O}(2)-\mathrm{N}(2)-\mathrm{C}(18)$ & $117.3(7)$ \\
\hline $\mathrm{C}(15)-\mathrm{N}(2)-\mathrm{C}(18)$ & $120.3(6)$ \\
\hline$C(3)-C(1)-N(1)$ & $107.9(7)$ \\
\hline$C(3)-C(1)-C(2)$ & $109.3(8)$ \\
\hline $\mathrm{N}(1)-\mathrm{C}(1)-\mathrm{C}(2)$ & $111.1(6)$ \\
\hline$C(3)-C(1)-C(4)$ & $109.6(6)$ \\
\hline $\mathrm{N}(1)-\mathrm{C}(1)-\mathrm{C}(4)$ & $108.2(7)$ \\
\hline$C(2)-C(1)-C(4)$ & $110.7(7)$ \\
\hline $\mathrm{C}(1)-\mathrm{C}(2)-\mathrm{H}(2 \mathrm{~A})$ & 109.5 \\
\hline $\mathrm{C}(1)-\mathrm{C}(2)-\mathrm{H}(2 \mathrm{~B})$ & 109.5 \\
\hline $\mathrm{H}(2 \mathrm{~A})-\mathrm{C}(2)-\mathrm{H}(2 \mathrm{~B})$ & 109.5 \\
\hline $\mathrm{C}(1)-\mathrm{C}(2)-\mathrm{H}(2 \mathrm{C})$ & 109.5 \\
\hline $\mathrm{H}(2 \mathrm{~A})-\mathrm{C}(2)-\mathrm{H}(2 \mathrm{C})$ & 109.5 \\
\hline $\mathrm{H}(2 \mathrm{~B})-\mathrm{C}(2)-\mathrm{H}(2 \mathrm{C})$ & 109.5 \\
\hline $\mathrm{C}(1)-\mathrm{C}(3)-\mathrm{H}(3 \mathrm{~A})$ & 109.5 \\
\hline $\mathrm{C}(1)-\mathrm{C}(3)-\mathrm{H}(3 \mathrm{~B})$ & 109.5 \\
\hline $\mathrm{H}(3 \mathrm{~A})-\mathrm{C}(3)-\mathrm{H}(3 \mathrm{~B})$ & 109.5 \\
\hline$C(1)-C(3)-H(3 C)$ & 109.5 \\
\hline $\mathrm{H}(3 \mathrm{~A})-\mathrm{C}(3)-\mathrm{H}(3 \mathrm{C})$ & 109.5 \\
\hline $\mathrm{H}(3 \mathrm{~B})-\mathrm{C}(3)-\mathrm{H}(3 \mathrm{C})$ & 109.5 \\
\hline$C(1)-C(4)-H(4 A)$ & 109.5 \\
\hline $\mathrm{C}(1)-\mathrm{C}(4)-\mathrm{H}(4 \mathrm{~B})$ & 109.5 \\
\hline $\mathrm{H}(4 \mathrm{~A})-\mathrm{C}(4)-\mathrm{H}(4 \mathrm{~B})$ & 109.5 \\
\hline $\mathrm{C}(1)-\mathrm{C}(4)-\mathrm{H}(4 \mathrm{C})$ & 109.5 \\
\hline $\mathrm{H}(4 \mathrm{~A})-\mathrm{C}(4)-\mathrm{H}(4 \mathrm{C})$ & 109.5 \\
\hline $\mathrm{H}(4 \mathrm{~B})-\mathrm{C}(4)-\mathrm{H}(4 \mathrm{C})$ & 109.5 \\
\hline$C(6)-C(5)-N(1)$ & $125.3(7)$ \\
\hline$C(6)-C(5)-C(10)$ & $118.5(9)$ \\
\hline$N(1)-C(5)-C(10)$ & $116.1(8)$ \\
\hline$C(7)-C(6)-C(5)$ & $120.7(8)$ \\
\hline$C(7)-C(6)-H(6 A)$ & 119.7 \\
\hline$C(5)-C(6)-H(6 A)$ & 119.7 \\
\hline$C(6)-C(7)-C(8)$ & $121.5(9)$ \\
\hline$C(6)-C(7)-H(7 A)$ & 119.2 \\
\hline $\mathrm{C}(8)-\mathrm{C}(7)-\mathrm{H}(7 \mathrm{~A})$ & 119.2 \\
\hline$C(9)-C(8)-C(7)$ & $117.4(8)$ \\
\hline$C(9)-C(8)-C(11)$ & $122.3(8)$ \\
\hline$C(7)-C(8)-C(11)$ & $119.9(8)$ \\
\hline$C(10)-C(9)-C(8)$ & $121.8(8)$ \\
\hline $\mathrm{C}(10)-\mathrm{C}(9)-\mathrm{H}(9 \mathrm{~A})$ & 119.1 \\
\hline$C(8)-C(9)-H(9 A)$ & 119.1 \\
\hline$C(9)-C(10)-C(5)$ & $120.0(9)$ \\
\hline $\mathrm{C}(9)-\mathrm{C}(10)-\mathrm{H}(10 \mathrm{~A})$ & 120.0 \\
\hline $\mathrm{C}(5)-\mathrm{C}(10)-\mathrm{H}(10 \mathrm{~A})$ & 120.0 \\
\hline$C(22)-C(11)-C(8)$ & $123.3(8)$ \\
\hline$C(22)-C(11)-C(12)$ & $123.1(9)$ \\
\hline$C(8)-C(11)-C(12)$ & $113.6(6)$ \\
\hline$C(17)-C(12)-C(13)$ & $118.6(7)$ \\
\hline$C(17)-C(12)-C(11)$ & $123.1(6)$ \\
\hline$C(13)-C(12)-C(11)$ & $117.8(8)$ \\
\hline$C(14)-C(13)-C(12)$ & $119.8(9)$ \\
\hline $\mathrm{C}(14)-\mathrm{C}(13)-\mathrm{H}(13 \mathrm{~A})$ & 120.1 \\
\hline $\mathrm{C}(12)-\mathrm{C}(13)-\mathrm{H}(13 \mathrm{~A})$ & 120.1 \\
\hline$C(15)-C(14)-C(13)$ & $120.7(7)$ \\
\hline $\mathrm{C}(15)-\mathrm{C}(14)-\mathrm{H}(14 \mathrm{~A})$ & 119.7 \\
\hline
\end{tabular}




\begin{tabular}{|c|c|}
\hline $\mathrm{C}(13)-\mathrm{C}(14)-\mathrm{H}(14 \mathrm{~A})$ & 119.7 \\
\hline$C(14)-C(15)-C(16)$ & $119.8(7)$ \\
\hline $\mathrm{C}(14)-\mathrm{C}(15)-\mathrm{N}(2)$ & $121.5(6)$ \\
\hline$C(16)-C(15)-N(2)$ & $118.3(8)$ \\
\hline$C(17)-C(16)-C(15)$ & $119.2(9)$ \\
\hline $\mathrm{C}(17)-\mathrm{C}(16)-\mathrm{H}(16 \mathrm{~A})$ & 120.4 \\
\hline$C(15)-C(16)-H(16 A)$ & 120.4 \\
\hline$C(12)-C(17)-C(16)$ & $121.6(6)$ \\
\hline $\mathrm{C}(12)-\mathrm{C}(17)-\mathrm{H}(17 \mathrm{~A})$ & 119.2 \\
\hline $\mathrm{C}(16)-\mathrm{C}(17)-\mathrm{H}(17 \mathrm{~A})$ & 119.2 \\
\hline $\mathrm{N}(2)-\mathrm{C}(18)-\mathrm{C}(20)$ & $107.9(8)$ \\
\hline$N(2)-C(18)-C(19)$ & $111.4(8)$ \\
\hline$C(20)-C(18)-C(19)$ & $110.5(6)$ \\
\hline $\mathrm{N}(2)-\mathrm{C}(18)-\mathrm{C}(21)$ & $106.7(6)$ \\
\hline$C(20)-C(18)-C(21)$ & $109.9(8)$ \\
\hline$C(19)-C(18)-C(21)$ & $110.3(7)$ \\
\hline $\mathrm{C}(18)-\mathrm{C}(19)-\mathrm{H}(19 \mathrm{~A})$ & 109.5 \\
\hline $\mathrm{C}(18)-\mathrm{C}(19)-\mathrm{H}(19 \mathrm{~B})$ & 109.5 \\
\hline $\mathrm{H}(19 \mathrm{~A})-\mathrm{C}(19)-\mathrm{H}(19 \mathrm{~B})$ & 109.5 \\
\hline $\mathrm{C}(18)-\mathrm{C}(19)-\mathrm{H}(19 \mathrm{C})$ & 109.5 \\
\hline $\mathrm{H}(19 \mathrm{~A})-\mathrm{C}(19)-\mathrm{H}(19 \mathrm{C})$ & 109.5 \\
\hline $\mathrm{H}(19 \mathrm{~B})-\mathrm{C}(19)-\mathrm{H}(19 \mathrm{C})$ & 109.5 \\
\hline $\mathrm{C}(18)-\mathrm{C}(20)-\mathrm{H}(20 \mathrm{~A})$ & 109.5 \\
\hline$C(18)-C(20)-H(20 B)$ & 109.5 \\
\hline $\mathrm{H}(20 \mathrm{~A})-\mathrm{C}(20)-\mathrm{H}(20 \mathrm{~B})$ & 109.5 \\
\hline $\mathrm{C}(18)-\mathrm{C}(20)-\mathrm{H}(20 \mathrm{C})$ & 109.5 \\
\hline $\mathrm{H}(20 \mathrm{~A})-\mathrm{C}(20)-\mathrm{H}(20 \mathrm{C})$ & 109.5 \\
\hline $\mathrm{H}(20 \mathrm{~B})-\mathrm{C}(20)-\mathrm{H}(20 \mathrm{C})$ & 109.5 \\
\hline$C(18)-C(21)-H(21 A)$ & 109.5 \\
\hline $\mathrm{C}(18)-\mathrm{C}(21)-\mathrm{H}(21 \mathrm{~B})$ & 109.5 \\
\hline $\mathrm{H}(21 \mathrm{~A})-\mathrm{C}(21)-\mathrm{H}(21 \mathrm{~B})$ & 109.5 \\
\hline $\mathrm{C}(18)-\mathrm{C}(21)-\mathrm{H}(21 \mathrm{C})$ & 109.5 \\
\hline $\mathrm{H}(21 \mathrm{~A})-\mathrm{C}(21)-\mathrm{H}(21 \mathrm{C})$ & 109.5 \\
\hline $\mathrm{H}(21 \mathrm{~B})-\mathrm{C}(21)-\mathrm{H}(21 \mathrm{C})$ & 109.5 \\
\hline$C(11)-C(22)-C(23)$ & $123.9(8)$ \\
\hline$C(11)-C(22)-C(27)$ & $122.9(9)$ \\
\hline$C(23)-C(22)-C(27)$ & $113.2(6)$ \\
\hline$C(22)-C(23)-C(31)$ & $108.7(8)$ \\
\hline$C(22)-C(23)-C(24)$ & $109.0(7)$ \\
\hline$C(31)-C(23)-C(24)$ & $109.1(5)$ \\
\hline$C(22)-C(23)-H(23 A)$ & 110.0 \\
\hline$C(31)-C(23)-H(23 A)$ & 110.0 \\
\hline$C(24)-C(23)-H(23 A)$ & 110.0 \\
\hline$C(25)-C(24)-C(23)$ & $108.4(7)$ \\
\hline$C(25)-C(24)-H(24 A)$ & 110.0 \\
\hline $\mathrm{C}(23)-\mathrm{C}(24)-\mathrm{H}(24 \mathrm{~A})$ & 110.0 \\
\hline$C(25)-C(24)-H(24 B)$ & 110.0 \\
\hline$C(23)-C(24)-H(24 B)$ & 110.0 \\
\hline $\mathrm{H}(24 \mathrm{~A})-\mathrm{C}(24)-\mathrm{H}(24 \mathrm{~B})$ & 108.4 \\
\hline$C(26)-C(25)-C(24)$ & $110.4(5)$ \\
\hline$C(26)-C(25)-C(30)$ & $109.9(8)$ \\
\hline$C(24)-C(25)-C(30)$ & $108.5(7)$ \\
\hline$C(26)-C(25)-H(25 A)$ & 109.3 \\
\hline$C(24)-C(25)-H(25 A)$ & 109.3 \\
\hline$C(30)-C(25)-H(25 A)$ & 109.3 \\
\hline$C(25)-C(26)-C(27)$ & $111.3(7)$ \\
\hline$C(25)-C(26)-H(26 A)$ & 109.4 \\
\hline$C(27)-C(26)-H(26 A)$ & 109.4 \\
\hline
\end{tabular}




\begin{tabular}{|c|c|}
\hline$C(25)-C(26)-H(26 B)$ & 109.4 \\
\hline$C(27)-C(26)-H(26 B)$ & 109.4 \\
\hline $\mathrm{H}(26 \mathrm{~A})-\mathrm{C}(26)-\mathrm{H}(26 \mathrm{~B})$ & 108.0 \\
\hline$C(22)-C(27)-C(28)$ & $108.8(7)$ \\
\hline$C(22)-C(27)-C(26)$ & $106.1(7)$ \\
\hline$C(28)-C(27)-C(26)$ & $108.7(5)$ \\
\hline$C(22)-C(27)-H(27 A)$ & 111.0 \\
\hline $\mathrm{C}(28)-\mathrm{C}(27)-\mathrm{H}(27 \mathrm{~A})$ & 111.0 \\
\hline$C(26)-C(27)-H(27 A)$ & 111.0 \\
\hline$C(29)-C(28)-C(27)$ & $110.8(8)$ \\
\hline $\mathrm{C}(29)-\mathrm{C}(28)-\mathrm{H}(28 \mathrm{~A})$ & 109.5 \\
\hline $\mathrm{C}(27)-\mathrm{C}(28)-\mathrm{H}(28 \mathrm{~A})$ & 109.5 \\
\hline $\mathrm{C}(29)-\mathrm{C}(28)-\mathrm{H}(28 \mathrm{~B})$ & 109.5 \\
\hline$C(27)-C(28)-H(28 B)$ & 109.5 \\
\hline $\mathrm{H}(28 \mathrm{~A})-\mathrm{C}(28)-\mathrm{H}(28 \mathrm{~B})$ & 108.1 \\
\hline$C(28)-C(29)-C(30)$ & $110.4(7)$ \\
\hline$C(28)-C(29)-C(31)$ & $109.4(6)$ \\
\hline$C(30)-C(29)-C(31)$ & $108.4(7)$ \\
\hline $\mathrm{C}(28)-\mathrm{C}(29)-\mathrm{H}(29 \mathrm{~A})$ & 109.6 \\
\hline$C(30)-C(29)-H(29 A)$ & 109.6 \\
\hline $\mathrm{C}(31)-\mathrm{C}(29)-\mathrm{H}(29 \mathrm{~A})$ & 109.6 \\
\hline$C(25)-C(30)-C(29)$ & $109.2(5)$ \\
\hline$C(25)-C(30)-H(30 A)$ & 109.8 \\
\hline $\mathrm{C}(29)-\mathrm{C}(30)-\mathrm{H}(30 \mathrm{~A})$ & 109.8 \\
\hline$C(25)-C(30)-H(30 B)$ & 109.8 \\
\hline$C(29)-C(30)-H(30 B)$ & 109.8 \\
\hline $\mathrm{H}(30 \mathrm{~A})-\mathrm{C}(30)-\mathrm{H}(30 \mathrm{~B})$ & 108.3 \\
\hline$C(23)-C(31)-C(29)$ & $110.1(7)$ \\
\hline $\mathrm{C}(23)-\mathrm{C}(31)-\mathrm{H}(31 \mathrm{~A})$ & 109.6 \\
\hline $\mathrm{C}(29)-\mathrm{C}(31)-\mathrm{H}(31 \mathrm{~A})$ & 109.6 \\
\hline $\mathrm{C}(23)-\mathrm{C}(31)-\mathrm{H}(31 \mathrm{~B})$ & 109.6 \\
\hline$C(29)-C(31)-H(31 B)$ & 109.6 \\
\hline $\mathrm{H}(31 \mathrm{~A})-\mathrm{C}(31)-\mathrm{H}(31 \mathrm{~B})$ & 108.2 \\
\hline
\end{tabular}

Table S24. Anisotropic displacement parameters $\left(\AA^{2} \times 10^{3}\right)$ for abcd3a $\left(\mathbf{3}(\mathbf{P h N i t})_{2}\right)$.

The anisotropic displacement factor exponent takes the form: $-2 \pi^{2}\left[h^{2} a^{* 2} U 11+\ldots+2 h k a^{*} b^{*} U 12\right]$

\begin{tabular}{lrrrrrr}
\hline U11 & U22 & U33 & U23 & U13 & U12 & \\
\hline $\mathrm{O}(1)$ & $12(4)$ & $11(5)$ & $18(3)$ & $1(2)$ & $-1(2)$ & $-5(3)$ \\
$\mathrm{O}(2)$ & $9(4)$ & $11(5)$ & $14(3)$ & $6(2)$ & $0(2)$ & $-6(3)$ \\
$\mathrm{N}(1)$ & $12(5)$ & $9(6)$ & $13(3)$ & $1(3)$ & $-2(3)$ & $-3(4)$ \\
$\mathrm{N}(2)$ & $15(5)$ & $22(7)$ & $6(3)$ & $1(3)$ & $-1(3)$ & $-10(4)$ \\
\hline
\end{tabular}

Table S25. Hydrogen coordinates ( x 10 $\left.0^{4}\right)$ and isotropic displacement parameters $\left(\AA^{2} \times 10^{3}\right)$ for abcd3a $\left(\mathbf{3}(\mathbf{P h N i t})_{2}\right)$.

\begin{tabular}{crrrr}
\hline \multicolumn{1}{c}{ X } & \multicolumn{2}{c}{ Z } & U (eq) & \\
\hline $\mathrm{H}(2 \mathrm{~A})$ & -20804 & -10184 & -8269 & 29 \\
$\mathrm{H}(2 \mathrm{~B})$ & -19132 & -10715 & -7740 & 29 \\
$\mathrm{H}(2 \mathrm{C})$ & -19126 & -9384 & -7625 & 29 \\
$\mathrm{H}(3 \mathrm{~A})$ & -23779 & -9251 & -7699 & 23 \\
$\mathrm{H}(3 \mathrm{~B})$ & -22147 & -8446 & -7041
\end{tabular}




\begin{tabular}{|c|c|c|c|c|}
\hline $\mathrm{H}(3 \mathrm{C})$ & -24039 & -9194 & -6810 & 23 \\
\hline $\mathrm{H}(4 \mathrm{~A})$ & -21395 & -10014 & -5936 & 23 \\
\hline $\mathrm{H}(4 \mathrm{~B})$ & -19490 & -9277 & -6170 & 23 \\
\hline $\mathrm{H}(4 \mathrm{C})$ & -19496 & -10608 & -6285 & 23 \\
\hline $\mathrm{H}(6 \mathrm{~A})$ & -19195 & -12054 & -7220 & 15 \\
\hline $\mathrm{H}(7 \mathrm{~A})$ & -18471 & -13911 & -7533 & 14 \\
\hline $\mathrm{H}(9 \mathrm{~A})$ & -24709 & -14889 & -8358 & 11 \\
\hline $\mathrm{H}(10 \mathrm{~A})$ & -25475 & -13035 & -8029 & 14 \\
\hline $\mathrm{H}(13 \mathrm{~A})$ & -21865 & -15613 & -6655 & 14 \\
\hline $\mathrm{H}(14 \mathrm{~A})$ & -19729 & -15800 & -5512 & 12 \\
\hline $\mathrm{H}(16 \mathrm{~A})$ & -15113 & -16879 & -6777 & 17 \\
\hline $\mathrm{H}(17 \mathrm{~A})$ & -17331 & -16803 & -7925 & 11 \\
\hline $\mathrm{H}(19 \mathrm{~A})$ & -18585 & -16458 & -4335 & 19 \\
\hline $\mathrm{H}(19 \mathrm{~B})$ & -18228 & -17711 & -4242 & 19 \\
\hline $\mathrm{H}(19 \mathrm{C})$ & -19067 & -17460 & -5069 & 19 \\
\hline $\mathrm{H}(20 \mathrm{~A})$ & -14815 & -15775 & -3745 & 19 \\
\hline $\mathrm{H}(20 \mathrm{~B})$ & -12904 & -16337 & -4109 & 19 \\
\hline $\mathrm{H}(20 \mathrm{C})$ & -14365 & -17011 & -3642 & 19 \\
\hline $\mathrm{H}(21 \mathrm{~A})$ & -13635 & -17972 & -5252 & 21 \\
\hline $\mathrm{H}(21 \mathrm{~B})$ & -16006 & -18399 & -5637 & 21 \\
\hline $\mathrm{H}(21 \mathrm{C})$ & -15165 & -18650 & -4810 & 21 \\
\hline $\mathrm{H}(23 \mathrm{~A})$ & -20945 & -18059 & -8425 & 13 \\
\hline $\mathrm{H}(24 \mathrm{~A})$ & -18446 & -17830 & -9337 & 14 \\
\hline $\mathrm{H}(24 \mathrm{~B})$ & -19809 & -19026 & -9629 & 14 \\
\hline $\mathrm{H}(25 \mathrm{~A})$ & -19712 & -18040 & -10699 & 12 \\
\hline $\mathrm{H}(26 \mathrm{~A})$ & -21337 & -16350 & -10673 & 14 \\
\hline $\mathrm{H}(26 \mathrm{~B})$ & -19452 & -16165 & -9974 & 14 \\
\hline $\mathrm{H}(27 \mathrm{~A})$ & -22549 & -15388 & -9494 & 12 \\
\hline $\mathrm{H}(28 \mathrm{~A})$ & -25679 & -16674 & -9537 & 16 \\
\hline $\mathrm{H}(28 \mathrm{~B})$ & -25205 & -16666 & -10399 & 16 \\
\hline $\mathrm{H}(29 \mathrm{~A})$ & -25958 & -18560 & -10241 & 16 \\
\hline $\mathrm{H}(30 \mathrm{~A})$ & -22851 & -19314 & -10711 & 15 \\
\hline $\mathrm{H}(30 \mathrm{~B})$ & -23461 & -18314 & -11138 & 15 \\
\hline $\mathrm{H}(31 \mathrm{~A})$ & -24678 & -18290 & -8871 & 11 \\
\hline $\mathrm{H}(31 \mathrm{~B})$ & -23617 & -19306 & -9331 & 11 \\
\hline
\end{tabular}

i SMART, Version 5.625, 2001, Bruker AXS Inc., Madison, Wisconsin, USA.

${ }^{i i}$ M. J. Hardie, K. Kirschbaum, A. Martin, A. A. Pinkerton J. Appl. Cryst. 1998, 31, 815-817.

iii Gemini 1.02 2000. Bruker AXS Inc., Madison, Wisconsin, USA.

${ }^{\text {iv }}$ Saint 6.29A, 2001 Bruker AXS Inc., Madison, Wisconsin, USA

v G.M. Sheldrick, SHELXTL, Version 5.1, 1998, Bruker AXS Inc., Madison, Wisconsin, USA. 